\title{
SEMI-CONTINUITY OF STABILITY FOR SHEAVES AND VARIATION OF GIESEKER MODULI SPACES
}

\author{
DANIEL GREB, JULIUS ROSS, AND MATEI TOMA
}

\begin{abstract}
We investigate a semi-continuity property for stability conditions for sheaves that is important for the problem of variation of the moduli spaces as the stability condition changes. We place this in the context of a notion of stability previously considered by the authors, called multi-Gieseker-stability, that generalises the classical notion of Giesekerstability to allow for several polarisations. As such we are able to prove that on smooth threefolds certain moduli spaces of Gieseker-stable sheaves are related by a finite number of Thaddeus-flips (that is flips arising for Variation of Geometric Invariant Theory) whose intermediate spaces are themselves moduli spaces of sheaves.
\end{abstract}

\section{INTRODUCTION}

The construction of moduli spaces of sheaves usually requires a choice of a stability condition, and in most cases different choices yield different spaces. The purpose of this paper is to investigate a "semi-continuity" property for such stability conditions which, as we shall see, plays an important role in the variation of these moduli spaces.

To begin the discussion, we somewhat vaguely denote a choice of stability condition by $\sigma$ which is allowed to vary in some space $\Sigma$. The best-known examples are slope-stability and Gieseker-stability, in which case $\sigma$ is a choice of (rational) ample line bundle and so $\Sigma$ is the ample cone of the base. More interesting examples arise from the stability conditions of Joyce [JII], that include the (twisted) multi-Gieseker stability previously considered by the authors [GRT14].

To discuss the variation problem, consider a path $\sigma(t) \in \Sigma$ for $t \in[0,1]$ that has a single "critical value" at the point $t=\bar{t}$. By this we mean that stability is unchanged as $t$ varies within either the interval $[0, \bar{t})$ or the interval $(\bar{t}, 1]$. Thus we are left with three moduli spaces that we denote by $\mathcal{M}_{\sigma(0)}, \mathcal{M}_{\sigma(\bar{t})}$ and $\mathcal{M}_{\sigma(1)}$ and we wish to understand how these are related. In the best possible case, there would exist a diagram of the form

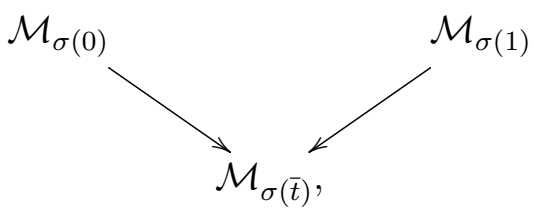

where the morphisms are induced from functorial properties of these spaces. For this to be possible in higher dimension, we immediately face two problems:

(1) (Existence of Moduli at Critical Values) We need that the moduli space $\mathcal{M}_{\sigma(\bar{t})}$ exists; that is we need to be able to construct the moduli spaces for critical values in $\Sigma$.

Date: April 25, 2016.

2010 Mathematics Subject Classification. 14D20, 14J60, 32G13; 14L24, 16G20.

Key words and phrases. Gieseker stability, variation of moduli spaces, chamber structures, moduli of quiver representations, semistable sheaves on Kähler manifolds. 
(2) (Semicontinuity) Assuming (1) holds, the existence of a diagram of the form $(*)$ requires that every sheaf that is semistable with respect to $\sigma(0)$ (resp. $\sigma(1)$ ) must also be semistable for $\sigma(\bar{t})$.

The existence of the moduli spaces at critical values can be problematic (on manifolds of dimension at least 3) for both slope-stability and Gieseker-stability, as the critical point in the ample cone at which stability changes may be irrational (see the discussion in [GRT14]). Furthermore semi-continuity will generally fail for stability conditions derived from a polynomial inequality (such as Gieseker-stability, see Remark 2.5)) that are natural from the viewpoint of algebraic geometry.

In this paper we study a notion of stability for sheaves which is restrictive enough to be able to produce moduli spaces at critical values (that are even projective) yet flexible enough to ensure that one can (conjecturally) always arrange so this semi-continuity holds. As will be discussed presently, we prove this conjecture in some particular cases, yielding an application to the variation problem for the moduli spaces of Gieseker stable sheaves.

Twisted Multi-Gieseker-stability. In previous work of the authors [GRT14] we introduce a notion of "multi-Gieseker stability" that extends the usual notion of Gieseker-stability of sheaves to the case of several polarisations, allowing us to study the variation problem in this context. For the purpose of this paper we require a slight generalisation that permits a fixed twisting of the Hilbert polynomials involved. To define this precisely, suppose that $L_{1}, \ldots, L_{j_{0}}$ are fixed ample line bundles on a smooth projective manifold $X$ and that $B_{1}, \ldots, B_{j_{0}}$ are a further collection of fixed line bundles. Given $\sigma_{1}, \ldots, \sigma_{j_{0}} \in \mathbb{R}_{>0}$, not all zero, we shall say a torsion-free coherent sheaf $E$ on $X$ is semistable with respect to this data if for all proper subsheaves $E^{\prime} \subset E$ the inequality

$$
\frac{\sum_{j} \sigma_{j} \chi\left(E^{\prime} \otimes L_{j}^{k} \otimes B_{j}\right)}{\operatorname{rank}\left(E^{\prime}\right)} \leq \frac{\sum_{j} \sigma_{j} \chi\left(E \otimes L_{j}^{k} \otimes B_{j}\right)}{\operatorname{rank}(E)}
$$

holds for all $k$ sufficiently large. We refer to $\sigma=\left(\sigma_{1}, \ldots, \sigma_{j_{0}}\right)$ as a stability parameter which we say is bounded if the set of semistable sheaves of a given topological type is bounded.

This definition generalises that of [GRT14] in which all the $B_{j}$ were taken to be trivial, and introduces no new difficulties so all the results from [GRT14] extend to this setting (see Section 1 for further discussion). In particular, semistable sheaves have projective moduli:

Theorem (Existence of projective moduli spaces of twisted semistable sheaves). Suppose $\sigma$ is a bounded stability parameter. Then, there exists a projective coarse moduli space $\mathcal{M}_{\sigma}$ of semistable sheaves of a given topological type. The moduli spaces $\mathcal{M}_{\sigma}$ contain an open set parameterizing stable sheaves, and the points on the boundary correspond to S-equivalence classes of sheaves.

A noteworthy aspect of this construction is that the critical values (or "walls") in $\Sigma:=$ $\left(\mathbb{R}_{>0}\right)^{j_{0}} \backslash\{0\}$ that witness the change of stability are given by rational linear functions. Thus there is no loss in assuming that all the $\sigma_{j}$ are rational, from which we conclude that the desired moduli spaces exist even at the critical values.

Hence we turn our attention to semi-continuity. Fixing the $L_{j}$ and $B_{j}$ as above, we say that a function $\sigma(t)=\left(\sigma_{1}(t), \ldots, \sigma_{j_{0}}(t)\right) \in \mathbb{R}_{\geq 0}^{j_{0}} \backslash\{0\}$ for $t \in[0,1]$ is a stability segment if each $\sigma_{j}$ is linear and

$$
\sum_{j} \operatorname{vol}\left(L_{j}\right) \sigma_{j}(t)=1 \quad \text { for all } t \in[0,1]
$$

where $\operatorname{vol}\left(L_{j}\right):=\int_{X} c_{1}\left(L_{j}\right)^{d}$ and $d=\operatorname{dim} X$. 
Definition (Uniform stability). We say a stability segment $(\sigma(t))_{t \in[0,1]}$ is uniform if for any torsion-free sheaf $E$ we have

$$
\frac{\sum_{j} \sigma_{j} \chi\left(E \otimes L_{j}^{k} \otimes B_{j}\right)}{\operatorname{rank}(E)}=\frac{k^{d}}{d !}+a_{d-1}(E) k^{d-1}+\cdots+a_{1}(E) k+a_{0}(E, t) \text { for } t \in[0,1]
$$

where $a_{d-1}(E), \ldots, a_{1}(E)$ are independent of $t$ and $a_{0}(E, t)$ is linear in $t$.

It is not hard to see that a uniform stability segment has the semi-continuity property (Remark 2.5). Roughly speaking then, the two main results of this paper say that uniform stability is (a) sufficiently strong to be able to control the variation of the moduli spaces as $t$ varies and (b) sufficiently flexible so that such segments can actually be constructed.

We discuss each of these in turn. For the first, by point of terminology, we say a stability segment $(\sigma(t))_{t \in[0,1]}$ is bounded if the set of sheaves of a given topological type that are semistable with respect to $\sigma(t)$ for some $t \in[0,1]$ is bounded. Throughout the paper by the topological type of a sheaf $E$ we shall mean its homological Todd class $\tau_{X}(E) \in B(X)_{\mathbb{Q}}:=$ $B(X) \otimes_{\mathbb{Z}} \mathbb{Q}$, where $B(X)$ is the group of cycles on $X$ modulo algebraic equivalence, see [GRT14, Def. 1.4].

Theorem. (Thaddeus-flips through moduli spaces of sheaves I, Theorem 2.6) Let X be smooth and projective, let $\tau \in B(X)_{\mathbb{Q}}$ and $(\sigma(t))_{t \in[0,1]}$ be a bounded uniform stability segment. Then given any $t^{\prime}<t^{\prime \prime}$ in $(0,1)^{1}$ the spaces $\mathcal{M}_{\sigma\left(t^{\prime}\right)}$ and $\mathcal{M}_{\sigma\left(t^{\prime \prime}\right)}$ are connected by a finite collection of Thaddeus-flips of the form

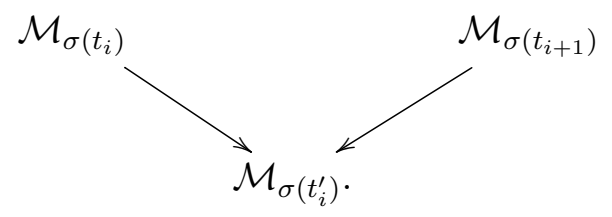

Thus uniform stability segments connect moduli spaces by Thaddeus-flips through moduli spaces of sheaves. Having established this result we will turn to the problem of constructing uniform stability segments. We show that on surfaces and threefolds this is possible, allowing us to conclude the following:

Theorem (Existence of Uniform Stability Segments, Theorem 6.1). Suppose $X$ is smooth and projective, let $\tau \in B(X)_{\mathbb{Q}}$, and $L^{\prime}, L^{\prime \prime} \in \operatorname{Amp}(X)$. Suppose also that either

(1) $X$ has dimension 2 or

(2) $X$ has dimension 3 and $L^{\prime}, L^{\prime \prime}$ are separated by a single wall of the first kind.

Then, the moduli spaces $\mathcal{M}_{L^{\prime}}$ and $\mathcal{M}_{L^{\prime \prime}}$ of Gieseker-semistable torsion-free sheaves of topological type $\tau$ are related by a finite number of Thaddeus-flips through spaces of the form $\mathcal{M}_{\sigma_{i}}$ for some bounded stability parameters $\sigma_{i}$.

The conclusion of this theorem for surfaces is precisely that of Matsuki-Wentworth [MW97], and our real interest lies in higher dimensions. We refer the reader to Definition 6.4 for the precise definition of being "separated by a single wall of the first kind", but we do not expect the hypotheses of this theorem to be optimal or particularly important. As will become apparent what we really discuss is a framework for producing uniform stability segments in general and we expect the following to be true:

\footnotetext{
${ }^{1}$ The assumption that $t^{\prime}$ and $t^{\prime \prime}$ lie in the open-interval $(0,1)$ will be relaxed in a sequel to include the endpoints.
} 
Conjecture. Any two bounded stability parameters $\sigma^{\prime}$ and $\sigma^{\prime \prime}$ can be joined by a bounded uniform stability segment,

If true one would conclude that any two moduli spaces $\mathcal{M}_{\sigma^{\prime}}$ and $\mathcal{M}_{\sigma^{\prime \prime}}$ are related by Thaddeus-flips through other moduli spaces of sheaves (in particular this would hold for any two moduli spaces of Gieseker semistable sheaves taken with respect to different polarisations). It seems likely that the above conjecture can be solved using our framework, but the details become much more involved.

Zooming. We briefly discuss the main idea used to construct uniform stability segments (see also Section 6.2 for a more detailed summary). Suppose that we have two bounded stability parameters $\sigma^{\prime}$ and $\sigma^{\prime \prime}$ (taken with respect to the same line bundles $L_{j}, B_{j}$ ) and we wish to show that the moduli spaces $\mathcal{M}_{\sigma^{\prime}}$ and $\mathcal{M}_{\sigma^{\prime \prime}}$ are related by Thaddeus-flips through moduli spaces of sheaves. The natural thing to do is to consider the straight line segment $(\sigma(t))_{t \in[0,1]}$ of stability parameters that joins $\sigma^{\prime}$ and $\sigma^{\prime \prime}$. If this segment were uniform, then we would be done by the discussion above, but in general this is not the case. Instead, there will be a finite number of rational "walls" that witness the change of multi-Gieseker-stability with respect to $\sigma(t)$ as $t$ varies in $[0,1]$. For simplicity, we assume these walls all lie in the interior $(0,1)$.

Now, the idea is to "zoom in" on a single one of these walls $\bar{t}$, and try to find another stability segment $(\eta(s))_{s \in[0,1]}$ for which the following holds:

Let $t_{-}$and $t_{+}$be points in $(0,1)$ immediately to the left and right of $\bar{t}$. Then, for $s>0$ sufficient small we have $\mathcal{M}_{\sigma\left(t_{-}\right)}=\mathcal{M}_{\eta(s)}$ and $\mathcal{M}_{\sigma\left(t_{+}\right)}=\mathcal{M}_{\eta(1-s)}$.

Now, if $\eta$ were uniform, then by the discussion above (Theorem 2.6) we get that $\mathcal{M}_{\sigma\left(t_{-}\right)}$ and $\mathcal{M}_{\sigma\left(t_{+}\right)}$are related by Thaddeus-flips though moduli spaces of sheaves. Thus, by applying this idea across each of the finitely many walls $\bar{t}$, we deduce the same holds for $\mathcal{M}_{\sigma^{\prime}}$ and $\mathcal{M}_{\sigma^{\prime \prime}}$.

It seems unlikely that one can always construct such an $\eta$ that is uniform. However, for surfaces and threefolds at least, we can construct such a segment that is "closer" to being uniform than $\sigma$ was. Roughly speaking, by this we mean that more coefficients of the relevant reduced multi-Hilbert polynomials associated with $\eta(s)$ are independent of $s$. We can then apply the same argument, and zoom-in on the walls for $\eta(s)$, and repeat this until eventually we are left with a chain of uniform stability segments, which join $\sigma^{\prime}$ and $\sigma^{\prime \prime}$.

Gieseker-stability with respect to real ample classes. As a by-product of the material we develop to define what it means for a polarisation to be general, cf. Section 4, we can give a statement about the existence and projectivity of moduli spaces of sheaves that are Gieseker-semistable with respect certain real classes $\omega \in \operatorname{Amp}(X)_{\mathbb{R}}$. We refer the reader to Section 3 for definitions of the walls $\tilde{W}_{i, F}$ used in the following statement.

Theorem (Projective moduli spaces for $\omega$-semistable sheaves, Theorem 5.1). Let $X$ be a smooth projective manifold of dimension d and $\tau \in B(X)_{\mathbb{Q}}$. Suppose that $K \subset \operatorname{Amp}(X)_{\mathbb{R}}$ is open and relatively compact and that $\omega \in \operatorname{Cone}^{*}(K)$ does not lie on any of the walls $\tilde{W}_{i, F}$ for $i \geq 2$ and $F \in \mathcal{S}_{K}$.

Then, there exists a projective moduli space $\mathcal{M}_{\omega}$ of torsion-free sheaves of topological type $\tau$ that are Gieseker-semistable with respect to $\omega$. This moduli space contains an open set consisting of points representing isomorphism classes of stable sheaves, while points on the boundary correspond to $S$-equivalence classes of strictly semistable sheaves.

We emphasise that the novelty of the previous Theorem is that $\omega$ is allowed to be real, possibly irrational, and we recall that for threefolds we proved more [GRT14, Theorem 11.6], 
namely that $\mathcal{M}_{\omega}$ is projective for all $\omega \in \operatorname{Amp}(X)_{\mathbb{R}}$. It would be interesting to know if this continues to hold for manifolds of any dimension.

Acknowledgements. The authors wish to thank Arend Bayer for helpful conversations at a crucial stage of this project. We also thank Dominic Joyce, Jun Li, and Alexander Schmitt for discussions comparing this work to theirs. In addition, we wish to thank Ivan Smith for discussions, and also acknowledge inspiration drawn from a talk by Aaron Bertram given in January 2014 at Banff International Research Station [Ber14]. JR is supported by an EPSRC Career Acceleration Fellowship (EP/J002062/1).

\section{Twisted multi-GiesekeR-Stability}

We start by extending the discussion of [GRT14] in two directions. In this section we allow a twisting of the stability condition by a collection of fixed line bundles, and in the next we make our main results uniform as the stability parameter varies.

Let $X$ be a projective manifold of dimension $d$. Suppose in addition to a vector $\underline{L}=$ $\left(L_{1}, \ldots, L_{j_{0}}\right)$ of ample line bundles we fix a vector $\underline{B}=\left(B_{1}, \ldots, B_{j_{0}}\right)$ of line bundles on $X$. Given a collection $\sigma_{1}, \ldots, \sigma_{j_{0}}$ of non-negative real numbers, not all zero, we define the multi-Hilbert polynomial with respect to this data of a torsion-free sheaf $E$ to be

$$
P_{E}^{\sigma}(m)=\sum_{j} \sigma_{j} \chi\left(E \otimes L_{j}^{m} \otimes B_{j}\right) .
$$

Writing this polynomial as $P_{E}^{\sigma}(m)=\sum_{i=0}^{d} \alpha_{i}^{\sigma}(E) \frac{m_{i}^{i}}{i !}$, we define the reduced multi-Hilbert polynomial as

$$
p_{E}^{\sigma}=\frac{P_{E}^{\sigma}}{\alpha_{d}^{\sigma}(E)} .
$$

We shall refer to either the data $\left(\underline{L}, \underline{B}, \sigma_{1}, \ldots, \sigma_{j_{0}}\right)$ or the vector $\left(\sigma_{1}, \ldots, \sigma_{j_{0}}\right)$ as a stability parameter.

Definition 1.1 (Multi-Gieseker-stability). We say that a torsion-free sheaf $E$ is multi-Gieseker(semi)stable, or just (semi)stable, if for all proper subsheaves $0 \neq F \subset E$ we have

$$
p_{F}^{\sigma}(\leq) p_{E}^{\sigma}
$$

Just as in the case of Gieseker-stability, any semistable sheaf admits a Jördan-Holder filtration, whose graded object is denoted $\operatorname{gr}(E)$. We say two semistable sheaves $E$ and $E^{\prime}$ are $S$-equivalent if $g r(E)$ and $g r\left(E^{\prime}\right)$ are isomorphic.

With these definitions at hand, all the results from [GRT14] extend to this twisted setting. More precisely, in this more general context the analogues of the Embedding Theorem, [GRT14, Theorem 5.6] (using the same quiver $Q$ ), and of the main "Comparison of semistability and JH filtrations" result, [GRT14, Theorem 8.1], hold. As a corollary of these results, there exist projective moduli spaces parametrising $S$-equivalence classes of $\sigma$ semistable sheaves of fixed topological type $\tau$, which we will denote by $\mathcal{M}_{\sigma}=\mathcal{M}_{\sigma}(\tau)$.

We do not repeat the proofs in this twisted setting, as no new ideas are needed and the existing proofs go through with essentially trivial modifications (and moreover many parts will actually follow from the work in the next section). The main change is that we now define

$$
T:=\bigoplus_{i j} L_{i}^{-n} \otimes B_{i}^{-1} \oplus L_{j}^{-m} \otimes B_{j}^{-1},
$$

so the quiver representation associated with a sheaf $E$ becomes

$$
\operatorname{Hom}(T, E):=\bigoplus_{i j} H^{0}\left(E \otimes L_{i}^{n} \otimes B_{i}\right) \oplus H^{0}\left(E \otimes L_{j}^{m} \otimes B_{j}\right)
$$


This is an $L \oplus H$ algebra, where $L$ is the algebra generated by the projection operators (precisely as in [GRT14, Section 5.1.2]) and where we adjust the definition of $H$ to be

$$
H:=\bigoplus_{i j} H_{i j}:=\bigoplus_{i j} H^{0}\left(X, L_{i}^{-n} \otimes L_{j}^{m} \otimes B_{i}^{-1} \otimes B_{j}\right) .
$$

To obtain the necessary regularity of the sheaves involved, we replace any instance of $(r, \underline{L})$ regularity with $(r, \underline{L}, \underline{B})$-regularity, which we define as follows:

Definition 1.2. We say a sheaf $E$ is $(r, \underline{L}, \underline{B})$-regular if $E \otimes B_{j}$ is $r$-regular with respect to $L_{j}$ for all $j=1, \ldots, j_{0}$.

We recall a sheaf $E^{\prime}$ is said to be $r$-regular with respect to a very ample line bundle $L$ if $H^{i}\left(E^{\prime} \otimes L^{r-i}\right)=0$ for all $i>0$. The above idea extends to the case that each $B_{j}$ is a formal sum of line bundles over $\mathbb{R}_{\geq 0}$, i.e., where for every $j=1, \ldots, j_{0}$, we have

$$
B_{j}=\sum_{i=1}^{N} b_{j i} B_{j i}
$$

where $B_{j i}$ is a line bundle, $b_{j i} \in \mathbb{R}_{\geq 0}$, and, to avoid trivialities, such that for all $j$ there is some $i$ with $b_{j i} \neq 0$. Given such data it is clear there is a (natural) stability parameter which we denote by $\sigma=\left(\underline{L} ; B_{1}, \ldots, B_{j_{0}}\right)$ with the property that for any sheaf $E$

$$
P_{E}^{\sigma}(k)=\sum_{j} \sum_{i} b_{j i} \chi\left(E \otimes L_{j}^{k} \otimes B_{j i}\right)
$$

Explicitly, one may take $\left(\underline{L} ; B_{1}, \ldots, B_{j_{0}}\right)$ to be

$$
\underbrace{\left(L_{1}, \ldots, L_{1}\right.}_{N \text { times }}, \ldots, \underbrace{L_{j_{0}}, \ldots, L_{j_{0}}}_{N \text { times }}, B_{11}, \ldots, B_{1 N}, \ldots, B_{j_{0} 1}, \ldots, B_{j_{0} N}, b_{11}, \ldots, b_{1 N}, \ldots, b_{j_{0} 1}, \ldots, b_{j_{0} N}) \text {. }
$$

Thus, in terms of the notation of [GRT14] we have $\left(\underline{L}, \sigma_{1}, \ldots, \sigma_{j_{0}}\right)=\left(\underline{L} ; \sigma_{1} \mathcal{O}_{X}, \ldots, \sigma_{j_{0}} \mathcal{O}_{X}\right)$.

Remark 1.3 (Riemann-Roch). We will frequently use the fact that such twisting does not affect the first non-trivial term of the reduced Hilbert polynomial. In detail, suppose $X$ is smooth of dimension $\operatorname{dim} X=d$, and given $\sigma=\left(\underline{L}, \underline{B}, \sigma_{1}, \ldots, \sigma_{j_{0}}\right)$ define

$$
\gamma:=\sum_{j} \sigma_{j} c_{1}\left(L_{j}\right)^{d-1} \in N_{1}(X)_{\mathbb{R}}
$$

(which we observe to be independent of $\underline{B}$ ). Then, using the Riemann-Roch theorem we have for any torsion-free sheaf $E$ on $X$ that

$$
p_{E}^{\sigma}=\frac{k^{d}}{d !}+\hat{\mu}^{\sigma}(E) \frac{k^{d-1}}{(d-1) !}+O\left(k^{d-2}\right),
$$

where

$$
\hat{\mu}^{\sigma}(E)=C_{1} \frac{\int_{X} c_{1}(E) \gamma}{\operatorname{rank} E}+C_{2}
$$

and $C_{1}, C_{2}$ are the topological constants

$$
C_{1}=\frac{1}{\sum_{j} \sigma_{j} \int_{X} c_{1}\left(L_{j}\right)^{d}} \text { and } C_{2}=\frac{\sum_{j} \sigma_{j} \int_{X}\left(\operatorname{Todd}_{1}(X)+c_{1}\left(B_{j}\right)\right) \cdot c_{1}\left(L_{j}\right)^{d-1}}{\sum_{j} \sigma_{j} \int_{X} c_{1}\left(L_{j}\right)^{d}},
$$

which are independent of $E$. In particular, precisely as in [GRT14, Lemma 3.3] for any torsion-free coherent sheaf $E$ the following implications hold:

slope stable with respect to $\gamma \Rightarrow$ stable with respect to $\sigma \Rightarrow$ semistable with respect to $\sigma \Rightarrow$ slope semistable with respect to $\gamma$. 
Consequently, any boundedness result for a set of sheaves of a given topological type that are slope semistable with respect to $\gamma$ implies boundedness for the set of sheaves of this topological type that are semistable with respect to $\sigma$.

\section{UNIFORMITY}

Let $X$ be a smooth variety of dimension $d$, fix $\underline{L}=\left(L_{1}, \ldots, L_{j_{0}}\right)$, where each $L_{j}$ is ample, and let $B_{1}, \ldots, B_{j_{0}}$ be line bundles. Moreover, let $\tau \in B(X)_{\mathbb{Q}}$, and set

$$
\operatorname{vol}\left(L_{j}\right):=\int_{X} c_{1}\left(L_{j}\right)^{d}
$$

We wish to consider the case of a segment of (twisted) stability parameters; that is for $t \in[0,1]$ let

$$
\sigma(t)=\left(\underline{L} ; \sigma_{1}(t) B_{1}, \ldots, \sigma_{j_{0}}(t) B_{j_{0}}\right) .
$$

Definition 2.1 (Stability segment). We say that $(\sigma(t))_{t \in[0,1]}$ is a stability segment if each $\sigma_{j}:[0,1] \rightarrow \mathbb{R}_{\geq 0}$ is a linear function, and if

$$
\sum_{j} \operatorname{vol}\left(L_{j}\right) \sigma_{j}(t)=1 \quad \text { for all } t \in[0,1] .
$$

Remark 2.2. Observe that since $\sigma_{j}(\cdot)$ is linear and non-negative, if $t \in(0,1)$ then $\sigma_{j}(t)>0$ for all $j$ so $\sigma(t)$ is a "positive" stability parameter in the language of [GRT14]. Also observe that in this case for any torsion-free sheaf $E$ the multiplicities are given by

$$
r_{E}^{\sigma(t)}=\sum_{j} \sigma_{j}(t) \operatorname{rank}(E) \operatorname{vol}\left(L_{j}\right)=\operatorname{rank}(E),
$$

and so the multi-Hilbert polynomial and reduced-Hilbert polynomial are related by

$$
p_{E}^{\sigma}(k)=\frac{P_{E}^{\sigma}(k)}{\operatorname{rank}(E)} .
$$

This is a polynomial in $k$ whose coefficients are linear functions of $t$.

It will be convenient later to allow the variation in $t$ to come from the twisting; so suppose $B_{j}(t)=\sum_{i} b_{j i}(t) B_{j i}$ for $j=1, \ldots, j_{0}$ is a formal sum of line bundles $B_{j i}$ whose coefficients $b_{j i}:[0,1] \rightarrow \mathbb{R}_{\geq 0}$ depend linearly on $t$. Then, the condition that

$$
\sigma(t)=\left(L_{1}, \ldots, L_{j_{0}} ; B_{1}(t), \ldots, B_{j_{0}}(t)\right)
$$

is a stability segment becomes

$$
\sum_{j} \operatorname{rank}\left(B_{j}(t)\right) \operatorname{vol}\left(L_{j}\right)=1 \quad \text { for all } t \in[0,1],
$$

where $\operatorname{rank}\left(B_{j}(t)\right):=\sum_{i} b_{j i}(t)$.

Definition 2.3 (Uniform stability segment). We say that a stability segment $(\sigma(t))_{t \in[0,1]}$ is uniform if the reduced multi-Hilbert polynomial of any torsion-free sheaf $E$ is of the form

$$
p_{E}^{\sigma(t)}(k)=\frac{k^{d}}{d !}+a_{d-1}(E) k^{d-1}+\cdots+a_{1}(E) k+a_{0}(E, t) \quad \text { for } t \in[0,1]
$$

where $a_{d-1}(E), \ldots, a_{1}(E)$ are independent of $t$ and $a_{0}(E, t)$ is linear in $t$.

The importance of the uniformity hypothesis stems from the following simple statement:

Lemma 2.4. Let $(\sigma(t))_{[0,1]}$ be a uniform stability segment, and let $E^{\prime} \subset E$ be torsion-free. 
(1) There is a $k_{0}$ such that for all $k \geq k_{0}$ and all $t \in[0,1]$ we have

$$
p_{E}^{\sigma(t)} \sim p_{E^{\prime}}^{\sigma(t)} \quad \text { if and only if } \quad p_{E}^{\sigma(t)}(k) \sim p_{E^{\prime}}^{\sigma(t)}(k),
$$

where $\sim$ is any of $\leq$ or $<$ or $\geq$ or $>$.

(2) For fixed $n, m$ the function $t \mapsto P_{E^{\prime}}^{\sigma(t)}(n) P_{E}^{\sigma(t)}(m)-P_{E}^{\sigma(t)}(n) P_{E^{\prime}}^{\sigma(t)}(m)$ is linear in $t$.

Proof. Using (2.2), we conclude the first statement directly from the uniformity hypothesis. For the second one, one observes that the same hypothesis implies the non-linear terms in $t$ cancel.

Remark 2.5. So, the crux of the above definition is that if $E^{\prime} \subset E$ is a proper subsheaf then

$$
p_{E}^{\sigma(t)}(k)-p_{E^{\prime}}^{\sigma(t)}(k)=\tilde{a}_{d-1} k^{d-1}+\cdots+\tilde{a}_{1} k+\tilde{a}_{0}(t),
$$

where only the constant term $\tilde{a}_{0}(t)$ depends non-trivially on $t$. This will certainly not hold in general, but below we will give a number of (natural) examples for which it does.

The property of being uniform gives semicontinuity of the chamber structure on $[0,1]$ defined by the stability parameters $\sigma(t)$. For suppose that a sheaf $E$ is semistable with respect to $\sigma\left(t_{p}\right)$ for a sequence $t_{p} \in[0,1]$ that have a limit $\bar{t}$ as $p$ tends to infinity. Then, without the uniformity assumption there is no reason to expect that $E$ is semistable with respect to $\sigma(\bar{t})$. To see this, observe that there could, in principle, be a subsheaf $E^{\prime} \subset E$ such that the difference of reduced multi-Hilbert polynomials is

$$
p_{E^{\prime}}^{\sigma(t)}(k)-p_{E}^{\sigma(t)}(k)=(t-\bar{t}) k+1
$$

in which case $E^{\prime}$ would destabilise $E$ for $t=\bar{t}$ but not for any $t<\bar{t}$.

However, under the assumptions of uniformity and boundedness it will be the case that $E$ is semistable with respect to $\sigma(\bar{t})$. To see this observe that we clearly need only consider saturated subsheaves $E^{\prime} \subset E$ with $\hat{\mu}^{\sigma(\bar{t})}\left(E^{\prime}\right) \geq \hat{\mu}^{\sigma(\bar{t})}(E)$ and these form a bounded family by Grothendieck's Lemma. Thus there are only a finite number of topological types of such $E^{\prime}$, and so we can find a $k_{0}$ so the conclusion of Lemma 2.4(1) holds for all such $E^{\prime}$. Thus semistability of $E$ with respect to $\sigma(t)$ for $t<\bar{t}$ implies $p_{E^{\prime}}^{\sigma(t)}\left(k_{0}\right) \leq p_{E}^{\sigma(t)}\left(k_{0}\right)$ for all $t<\bar{t}$, and thus by continuity $p_{E^{\prime}}^{\sigma(\bar{t})}\left(k_{0}\right) \leq p_{E}^{\sigma(\bar{t})}\left(k_{0}\right)$ as well. So applying the Lemma again we get $p_{E^{\prime}}^{\sigma(\bar{t})} \leq p_{E}^{\sigma(\bar{t})}$ and since this holds for all these subsheaves we conclude $E$ is semistable with respect to $\sigma(\bar{t})$ as well.

We say that a stability segment $(\sigma(t))_{t \in[0,1]}$ is bounded if the set of sheaves $E$ of topological type $\tau$ that are semistable with respect to $\sigma(t)$ for some $t \in[0,1]$ is bounded. Our goal is to prove the following:

Theorem 2.6. (Thaddeus-Flips Through Moduli Space of Sheaves) Let X be smooth, projective and $\tau \in B(X)_{\mathbb{Q}}$. Suppose $\sigma(t)_{t \in[0,1]}$ is a bounded and uniform segment of stability parameters and let $t^{\prime}, t^{\prime \prime} \in(0,1) \cap \mathbb{Q}$ with $t^{\prime}<t^{\prime \prime}$. Then there exists a finite sequence of rational numbers $t^{\prime}=t_{0}<t_{1}<\ldots<t_{N}=t^{\prime \prime}$ so that for $i=0, \ldots, N-1$ there exist Thaddeus-flips

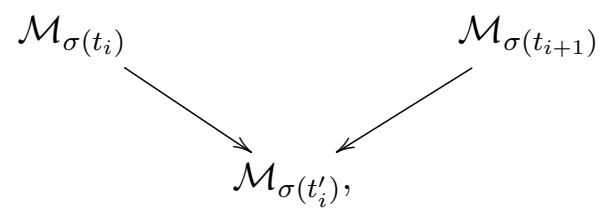

where $t_{i}^{\prime} \in\left(t_{i}, t_{i+1}\right)$. Thus, $\mathcal{M}_{\sigma\left(t^{\prime}\right)}$ and $\mathcal{M}_{\sigma\left(t^{\prime \prime}\right)}$ are related by a finite number of Thaddeus-flips through spaces of moduli spaces of sheaves. 
2.1. Uniform Version of the Le Potier-Simpson Theorem. One of the main technical results of [GRT14] states if $\sigma$ is a given bounded stability parameter, then for $m \gg n \gg p \gg 0$ a sheaf $E$ (of a given topological type $\tau$ ) is semistable if and only if the corresponding module $\operatorname{Hom}(T, E)$ is semistable [GRT14, Theorem 8.1]. We now enhance this result by showing that if $(\sigma(t))_{t \in[0,1]}$ is a bounded and uniform segment of stability conditions then one can choose $m, n, p$ uniformly over all $t \in[0,1]$.

To simplify the discussion, we will say a sheaf $E$ is semistable with respect to $t \in[0,1]$ if it is semistable with respect to $\sigma(t)$, and we let $P_{E}^{t}$ and $p_{E}^{t}$ denote the corresponding multi-Hilbert polynomial and reduced multi-Hilbert polynomial of $E$, respectively. By the boundedness hypothesis we may pick $p$ such that any sheaf $E$ of type $\tau$ that is semistable with respect to some $t \in[0,1]$ is $(p, \underline{L}, \underline{B})$-regular in the sense of Definition 1.2.

Theorem 2.7 (Uniform version of the Le Potier-Simpson Theorem). Let $X$ be a smooth projective variety, and suppose $(\sigma(t))_{t \in[0,1]}$ is a bounded and uniform segment of stability parameters. Then, if $n \gg p$, for any torsion-free sheaf $E$ of topological type $\tau$ and any $t \in[0,1]$ the following are equivalent:

(1) $E$ is (semi)stable with respect to $t$.

(2) $E$ is $(p, \underline{L}, \underline{B})$-regular and for all proper $E^{\prime} \subset E$ we have

$$
\frac{\sum_{j} \sigma_{j}(t) h^{0}\left(E^{\prime} \otimes L_{j}^{n} \otimes B_{j}\right)}{\operatorname{rank}\left(E^{\prime}\right)}(\leq) p_{E}^{t}(n) .
$$

Moreover, if the saturation of $E^{\prime}$ in $E$ is not $(n, \underline{L}, \underline{B})$-regular, then

$$
\frac{\sum_{j} \sigma_{j}(t) h^{0}\left(E^{\prime} \otimes L_{j}^{n} \otimes B_{j}\right)}{\operatorname{rank}\left(E^{\prime}\right)} \leq p_{E}^{t}(n)-1 .
$$

(3) $E$ is $(p, \underline{L}, \underline{B})$-regular and for all proper saturated $E^{\prime} \subset E$ with $\hat{\mu}^{\sigma(t)}\left(E^{\prime}\right) \geq \hat{\mu}^{\sigma(t)}(E)$ the inequality (2.3) holds.

Moreover, if $E$ is semistable of topological type $\tau$, and $E^{\prime} \subset E$ is a proper subsheaf, then equality holds in (2.3) if and only if $E^{\prime}$ is destabilising.

Proof. This is essentially the same as the proof for a single stability parameter. Choose $\bar{C}$ and $C_{1}$, as in the proof of [GRT14, Theorem 7.2], and then choose $C_{2}$ so that for any $(p, \underline{L}, \underline{B})$-regular sheaf $E$ of type $\tau$ and any $t \in[0,1]$ we have

$$
C_{2} \geq-\hat{\mu}^{\sigma(t)}(E)+1
$$

and such that additionally

$$
\left(1-\frac{\sum_{j} \sigma_{j}(t)}{\operatorname{rank}(E)}\right)\left(C_{1}+\bar{C}\right)+\frac{\sum_{j} \sigma_{j}(t)}{\operatorname{rank}(E)}\left(-C_{2}+\bar{C}\right) \leq \hat{\mu}^{\sigma(t)}(E)-1
$$

holds for all $t \in[0,1]$. Then, let $\mathcal{S}$ be the set of all saturated subsheaves $F \subset E$ where $E$ is $(p, \underline{L}, \underline{B})$-regular and $\hat{\mu}^{L_{j}}(F) \geq-C_{2}$ for some $1 \leq j \leq j_{0}$. This set is bounded, and so for $n \gg p$ we can arrange the conditions (i)-(iv) from that proof to hold uniformly over $t \in[0,1]$. The condition (i) can be achieved by Lemma 2.4 since there are a finite number of different $p_{F}$ as $F$ ranges over the bounded set $\mathcal{S}$, and condition (iv) holds uniformly over $t \in[0,1]$, since (2.6) implies the leading order term in the polynomial on the left hand side of (iv) is less than or equal to the leading term in the right hand side minus 1; thus, the inequality (iv) holds for all $n$ sufficiently large. 
From this point on, the proof is the same as that of [GRT14, Theorem 7.2], noting that if $E$ is semistable and $E^{\prime} \subset E$ is a sheaf whose saturation is not $(n, \underline{L}, \underline{B})$-regular then it is necessarily not of type (B) and thus the stronger inequality (2.4) holds.

Remark 2.8. As is clear from the proof of the (3) implies (1) direction, we actually have that with $n$ as chosen above, if $E$ is pure of dimension $d$ of type $\tau$ and $(p, \underline{L}, \underline{B})$-regular, and $E^{\prime} \subset E$ is saturated and $(n, \underline{L}, \underline{B})$-regular with

$$
\frac{\sum_{j} \sigma_{j} h^{0}\left(E^{\prime} \otimes L_{j}^{n}\right)}{r_{E^{\prime}}^{\sigma}} \leq p_{E}^{\sigma}(n)
$$

then $p_{E^{\prime}}^{\sigma} \leq p_{E}^{\sigma}$.

2.2. Uniform Comparison of Semistability. We continue as above, so $X$ is smooth and projective of dimension $d$ and we fixed a class $\tau \in B(X)_{\mathbb{Q}}$.

Theorem 2.9 (Uniform comparison of semistability and JH filtrations). Let $(\sigma(t))_{t \in[0,1]}$ be a bounded and uniform segment of stability parameters and let $t^{\prime}, t^{\prime \prime} \in(0,1) \cap \mathbb{Q}$ with $t^{\prime}<t^{\prime \prime}$. Then, for $m \gg n \gg p \gg 0$ and all $t \in\left[t^{\prime}, t^{\prime \prime}\right]$ the following holds for any sheaf $E$ on $X$ of topological type $\tau$ :

(1) $E$ is semistable with respect to $\sigma(t)$ if and only if it is torsion free, $(p, \underline{L}, \underline{B})$-regular, and $\operatorname{Hom}(T, E)$ is semistable (in the sense of representations of a quiver).

(2) If $E$ is semistable, then

$$
\operatorname{Hom}(T, g r E) \simeq g r \operatorname{Hom}(T, E),
$$

where gr denotes the graded object coming from a Jordan-Hölder filtration of $E$ or $\operatorname{Hom}(T, E)$, respectively (taken with respect to $\sigma(t)$ ). In particular, two semistable sheaves $E$ and $E^{\prime}$ are $S$-equivalent if and only if $\operatorname{Hom}(T, E)$ and $\operatorname{Hom}\left(T, E^{\prime}\right)$ are $S$ equivalent.

For the proof we first make explicit our choice of integers. First, we use boundedness to choose $p$ large enough so that

(C1') Any sheaf $E$ of topological type $\tau$ that is semistable with respect to some $t \in[0,1]$ is $(p, \underline{L}, \underline{B})$-regular.

Now, let $\mathcal{S}_{2}$ be the set of saturated subsheaves $E^{\prime} \subset E$ where $E$ is $(p, \underline{L}, \underline{B})$-regular of topological type $\tau$ and $\hat{\mu}^{\sigma(t)}\left(E^{\prime}\right) \geq \hat{\mu}^{\sigma(t)}(E)$ for some $t \in[0,1]$. Then, $\mathcal{S}_{2}$ is bounded by Lemma 3.1 below. So, we may let $n$ be large enough so

(C2') (a) The conclusion of the Uniform Version of the Le Potier-Simpson Theorem (Theorem 2.7) holds and (b) any sheaf $E^{\prime} \subset \mathcal{S}_{2}$ is $(n, \underline{L}, \underline{B})$-regular.

Now, as in [GRT14, Section 4], the interval [0,1] admits a finite chamber decomposition that witnesses the change of stability as $t$ varies. In detail, this says there exists a finite sequence $t^{\prime}=t_{0}<t_{1}<\ldots<t_{N}=t^{\prime \prime}$ of rational numbers such that any if $E$ is any $(p, \underline{L}, \underline{B})$ regular sheaf and $E^{\prime} \subset E$ is in $\mathcal{S}_{2}$, then $p_{E^{\prime}}^{t} \leq p_{E}^{t}$ for some $t \in\left(t_{i}, t_{i+1}\right)$ implies that this holds for all $t \in\left(t_{i}, t_{i+1}\right)$. In particular, if $E$ is semistable with respect to $t \in\left(t_{i}, t_{i+1}\right)$, then it is semistable with respect to all $t \in\left(t_{i}, t_{i+1}\right)$, and thus by uniformity, Remark 2.5 , we know that if this holds, then in fact $E$ is semistable with respect to $t_{i}$ and $t_{i+1}$ as well.

For each $E^{\prime} \subset E$ where $E$ is $(p, \underline{L}, \underline{B})$-regular of topological type $\tau$ and $E^{\prime} \in \mathcal{S}_{2}$, consider the function

$$
f_{E^{\prime}}(t)=P_{E^{\prime}}^{t}(n) P_{E}^{t}(m)-P_{E}^{t}(n) P_{E^{\prime}}^{t}(m) .
$$


By boundedness we may enlarge the set $\left\{t_{0}, \ldots, t_{N}\right\}$ and assume that for all such $E^{\prime} \subset E$ the following holds:

$$
\text { if } f_{E^{\prime}} \not \equiv 0 \text {, then } f_{E^{\prime}}(t)=0 \text { for some } t \in\left[t^{\prime}, t^{\prime \prime}\right] \text { implies } t=t_{i} \text { for some } i \text {. }
$$

We now choose $m \gg n$ large enough so that

(C3') Each $L_{j}^{-n}$ is $(m, \underline{L}, \underline{B})$-regular.

For the next condition we make a construction completely analogous to that of [GRT14, Section 8]. Let $E$ be any sheaf that is $(n, \underline{L}, \underline{B})$-regular and has topological type $\tau$. For each $j$ let

$$
\epsilon_{j}: H^{0}\left(E \otimes L_{j}^{n} \otimes B_{j}\right) \otimes L_{j}^{-n} \otimes B_{j}^{-1} \rightarrow E
$$

be the natural (surjective) evaluation maps.

Definition 2.10. For an $(n, \underline{L}, \underline{B})$-regular sheaf $E$ and subspaces $V_{j}^{\prime} \subset H^{0}\left(E \otimes L_{j}^{n} \otimes B_{j}\right)$ let $E_{j}^{\prime}$ and $F_{j}^{\prime}$ be the image and kernel of $\epsilon_{j}$ restricted to $V_{j}^{\prime}$, so there is a short exact sequence $0 \rightarrow F_{j}^{\prime} \rightarrow V_{j}^{\prime} \otimes L_{j}^{-n} \rightarrow E_{j}^{\prime} \rightarrow 0$. Then, define a subsheaf of $E$ by

$$
E_{\text {sum }}:=E_{\text {sum }}\left(V_{1}^{\prime}, \ldots, V_{j_{0}}^{\prime}\right):=E_{1}^{\prime}+\cdots+E_{j_{0}}^{\prime}
$$

and let $K=K\left(V_{1}, \ldots, V_{j_{0}}\right)$ be the kernel of the surjection $\bigoplus_{j} E_{j}^{\prime} \rightarrow E_{\text {sum. }}$. We let $\mathcal{S}_{1}$ be the bounded set of all sheaves $E_{j}^{\prime}, F_{j}^{\prime}, E_{\text {sum }}$ and $K$ that arise in this way.

By increasing $m$ if necessary, we may assume the following.

(C4') All the sheaves in set $\mathcal{S}_{1}$ are $(m, \underline{L}, \underline{B})$-regular.

Note the assumption (C2')(a) implies the usual (non-uniform) Le Potier-Simpson Theorem holds [GRT14, Theorem 7.2]. Since by $\left(\mathrm{C}^{\prime}\right)(\mathrm{b})$ any sheaf in $\mathcal{S}_{2}$ is certainly $(m, \underline{L}, \underline{B})$ regular, we see that the conditions so far imply the (twisted versions of) conditions (C1)-(C4) from [GRT14, Section 8] hold for each $t \in\left[t^{\prime}, t^{\prime \prime}\right]$.

The analogue of condition (C5) is more subtle, for this we require the following:

(C5') For each $i$, let $t_{i}^{\prime}$ be a point in the interval $\left(t_{i}, t_{i+1}\right)$. Then, for any $t \in\left\{t_{i}, t_{i}^{\prime}\right\}$ the twisted version of condition (C5) from [GRT14, Section 8] holds. That is, if $P_{j}(k)=\chi\left(E \otimes L_{j}^{k} \otimes B_{j}\right)$, then for any integers $c_{j} \in\left\{0, \ldots, P_{j}(n)\right\}$ and sheaves $E^{\prime} \in \mathcal{S}_{1} \cup \mathcal{S}_{2}$ the polynomial relation $P_{E}^{t}\left(\sum_{j} \sigma_{j}^{t}\right) c_{j} \sim P_{E^{\prime}}^{t} P_{E}^{t}(n)$ is equivalent to the relation $P_{E}^{t}(m) \sum_{j} \sigma_{j}(t) c_{j} \sim P_{E^{\prime}}^{t}(m) P_{E}^{t}(n)$, where $\sim$ is any of $\leq$ or $<$ or $=$.

We note that $\left(\mathrm{C} 5^{\prime}\right)$ is possible for the same reason that (C5) was possible, since we are only demanding that it hold for a finite number of stability parameters. Thus, we have that (the twisted versions of) conditions (C1) though (C5) holds for all the $\sigma\left(t_{i}\right)$ and $\sigma\left(t_{i}^{\prime}\right)$. In particular, the proof given in [GRT14, Section 8] applies to establish the Comparison of Semistability and of JH filtrations, [GRT14, Theorem 8.1], at these finite collection of points, giving the following:

Theorem 2.11. The conclusion of Theorem 2.9 holds if $t$ is contained in the finite set $\left\{t_{i}, t_{i}^{\prime}\right\}$.

We next claim that by enlarging $m$ if necessary, we may also assume the following holds for all $t \in[0,1]$ :

(C6') If $t \in[0,1]$ and $E^{\prime} \subset E$ with $E(p, \underline{L}, \underline{B})$-regular of topological type $\tau$ and $E^{\prime} \in \mathcal{S}_{1}$ is such that

$$
\sum_{j} \sigma_{j}(t) h^{0}\left(E^{\prime} \otimes L_{j}^{n} \otimes B_{j}\right) \operatorname{rank}(E) \leq P_{E}^{t}(n) \operatorname{rank}\left(E^{\prime}\right)-\frac{1}{\max _{j} \operatorname{vol}\left(L_{j}\right)},
$$


then

$$
\sum_{j} \sigma_{j}(t) h^{0}\left(E^{\prime} \otimes L_{j}^{n} \otimes B_{j}\right) P_{E}^{t}(m) \leq P_{E}^{t}(n) P_{E^{\prime}}^{t}(m)-1 .
$$

To see this, observe that as $\mathcal{S}_{1}$ is bounded, the set of multi-Hilbert polynomials $P_{E^{\prime}}^{\sigma(t)}$ that arise for $E^{\prime} \in \mathcal{S}_{1}$ is finite. Now, (2.8) says that the corresponding inequality in the leading order coefficient in $m$ (namely the coefficient of $m^{d}$ ) in (2.9) holds strictly (and by an amount bounded away from 0 for all $t \in[0,1]$ ). So, as the coefficients of all the lower order terms are bounded over $t \in[0,1]$ for all such $E^{\prime}$, we conclude that (2.9) holds for all $m \gg 0$.

Having made our choice of integers $m, n, p$ we turn to some preliminary lemmas needed for our proof of the Uniform Comparison of Semistability. For a non-trivial $A$-module $M=$ $\bigoplus_{j} V_{j} \oplus W_{j}$ we set

$$
\mu_{t}(M)=\frac{\sum_{j} \sigma_{j}(t) \operatorname{dim} V_{j}}{\sum_{j} \sigma_{j}(t) \operatorname{dim} W_{j}} \quad \text { for } t \in(0,1) .
$$

Moreover, we recall the following technical definition from [GRT14].

Definition 2.12. Let $M^{\prime}=\bigoplus_{j} V_{j}^{\prime} \oplus W_{j}^{\prime}$ and $M^{\prime \prime}=\bigoplus_{j} V_{j}^{\prime \prime} \oplus W_{j}^{\prime \prime}$ be two submodules of a given $A$-module $M$. We say that $M^{\prime}$ is subordinate to $M^{\prime \prime}$ if

$$
V_{j}^{\prime} \subset V_{j}^{\prime \prime} \text { and } W_{j}^{\prime \prime} \subset W_{j}^{\prime} \quad \text { for all } j \text {. }
$$

Lemma 2.13. Let $E$ be a torsion-free sheaf of type $\tau$. Suppose $t \in(0,1)$ is such that $E$ is semistable with respect $\sigma(t)$. Then, if $M^{\prime}$ is a submodule of $M=\operatorname{Hom}(T, E)$ with $\mu_{t}\left(M^{\prime}\right)=\mu_{t}(M)$, there exists an $(n, \underline{L}, \underline{B})$-regular sheaf $E^{\prime} \subset E$ such that $M^{\prime}$ is subordinate to $\operatorname{Hom}\left(T, E^{\prime}\right)$.

Proof. Write $M^{\prime}=\oplus_{j} V_{j}^{\prime} \oplus W_{j}^{\prime}$ and $E^{\prime}=E_{\text {sum }}\left(V_{1}^{\prime}, \ldots, V_{j_{0}}^{\prime}\right)$. From [GRT14, Proposition 8.13] we know that $M^{\prime}$ is subordinate to $\operatorname{Hom}\left(T, E^{\prime}\right)$. So, the issue is to prove that $E^{\prime}$ is $(n, \underline{L}, \underline{B})$-regular. To this end, we first claim that

$$
\sum_{j} \sigma_{j}(t) h^{0}\left(E^{\prime} \otimes L_{j}^{n} \otimes B_{j}\right) \operatorname{rank}(E)>P_{E}^{t}(n) \operatorname{rank}\left(E^{\prime}\right)-\frac{1}{\max _{j} \operatorname{vol}\left(L_{j}\right)} .
$$

To see this, suppose for contradiction that in fact

$$
\sum_{j} \sigma_{j}(t) h^{0}\left(E^{\prime} \otimes L_{j}^{n} \otimes B_{j}\right) \operatorname{rank}(E) \leq P_{E}^{t}(n) \operatorname{rank}\left(E^{\prime}\right)-\frac{1}{\max _{j} \operatorname{vol}\left(L_{j}\right)} .
$$

By (C4') we know $E^{\prime}$ is $(m, \underline{L}, \underline{B})$-regular, and so using condition (C6') we have

$$
\begin{aligned}
\mu_{t}\left(\operatorname{Hom}\left(T, E^{\prime}\right)\right)=\frac{\sum_{j} \sigma_{j}(t) h^{0}\left(E^{\prime} \otimes L_{j}^{n} \otimes B_{j}\right)}{P_{E^{\prime}}^{t}(m)} & \leq \frac{P_{E}^{t}(n)}{P_{E}^{t}(m)}-\frac{1}{P_{E}^{t}(m) P_{E^{\prime}}^{t}(m)} \\
& <\frac{P_{E}^{t}(n)}{P_{E}^{t}(m)}=\mu_{t}(M) .
\end{aligned}
$$

On the other hand $M^{\prime}$ is subordinate to $\operatorname{Hom}\left(T, E^{\prime}\right)$, so certainly $\mu_{t}\left(\operatorname{Hom}\left(T, E^{\prime}\right)\right) \geq \mu_{t}\left(M^{\prime}\right)=$ $\mu_{t}(M)$ which gives the desired contradiction.

Thus, by the assumption that $E$ is semistable and by part a) of (C2') the "(1) implies (2)" direction of the Uniform Le Potier-Simpson Theorem (Theorem (2.7)) holds, so we know that the saturation $F$ of $E^{\prime}$ is $(n, \underline{L}, \underline{B})$-regular. So, it is sufficient to prove that $E^{\prime}$ is saturated.

To this end, observe that if $h^{0}\left(E^{\prime} \otimes L_{j}^{n} \otimes B_{j}\right)=h^{0}\left(F \otimes L_{j}^{n} \otimes B_{j}\right)$ for some $j$, then $F=E^{\prime}$ as $F \otimes B_{j} \otimes L_{j}^{n}$ is globally generated, and so we are done. So, suppose this is not the case, 
i.e., $h^{0}\left(E^{\prime} \otimes L_{j}^{n} \otimes B_{j}\right) \leq h^{0}\left(F \otimes L_{j}^{n} \otimes B_{j}\right)-1$ for all $j$. Then, using $\operatorname{rank}\left(E^{\prime}\right)=\operatorname{rank}(F)$ we obtain

$$
\frac{\sum_{j} \sigma_{j}(t) h^{0}\left(E^{\prime} \otimes L_{j}^{n} \otimes B_{j}\right)}{\operatorname{rank}\left(E^{\prime}\right)} \leq \frac{\sum \sigma_{j}(t) h^{0}\left(F \otimes L_{j}^{n} \otimes B_{j}\right)}{\operatorname{rank}(F)}-\frac{\sum_{j} \sigma_{j}(t)}{\operatorname{rank}(F)}
$$

As $E$ is semistable, we can again apply the "(1) implies (2)" direction of the Le Potier-Simpson (Theorem 2.7) to give that the right hand side of the previous equation is in turn bounded by

$$
\frac{P_{E}^{t}(n)}{\operatorname{rank}(E)}-\frac{\sum_{j} \sigma_{j}(t)}{\operatorname{rank}(F)}=\frac{P_{E}^{t}(n)}{\operatorname{rank}(E)}-\frac{\sum_{j} \sigma_{j}(t)}{\operatorname{rank}\left(E^{\prime}\right)}
$$

and so

$$
\begin{aligned}
\sum_{j} \sigma_{j}(t) h^{0}\left(E^{\prime} \otimes L_{j}^{n} \otimes B_{j}\right) \operatorname{rank}(E) & \leq P_{E}^{t}(n) \operatorname{rank}\left(E^{\prime}\right)-\sum_{j} \sigma_{j}(t) \operatorname{rank}(E) \\
& \leq P_{E}^{t}(n) \operatorname{rank}\left(E^{\prime}\right)-\frac{1}{\max _{j} \operatorname{vol}\left(L_{j}\right)}
\end{aligned}
$$

as $\sum_{j} \sigma_{j}(t) \cdot \max _{j} \operatorname{vol}\left(L_{j}\right) \geq \sum_{j} \sigma_{j}(t) \operatorname{vol}\left(L_{j}\right)=1$. This contradicts the above claim and therefore completes the proof.

The next lemma is a slight refinement of our Comparison of Semistability Theorem for a single stability parameter, which could just as well have been proved in [GRT14, Section 8].

Lemma 2.14. Let $t=t_{i}$ for some $i$. Suppose that $E$ is $(p, \underline{L}, \underline{B})$-regular of topological type $\tau$ and that $E^{\prime} \subset E$ has $p_{E^{\prime}}^{t}>p_{E}^{t}$. Then, $\mu_{t}\left(\operatorname{Hom}\left(T, E^{\prime}\right)\right)>\mu_{t}(\operatorname{Hom}(T, E))$.

Proof. We have $\hat{\mu}_{E^{\prime}}^{t_{i}} \geq \hat{\mu}_{E}^{t_{i}}$ and so $E^{\prime} \in \mathcal{S}_{2}$, which implies $E^{\prime}$ is $(n, \underline{L}, \underline{B})$-regular by (C2'). Suppose for contradiction $\mu_{t}\left(\operatorname{Hom}\left(T, E^{\prime}\right)\right) \leq \mu_{t}(\operatorname{Hom}(T, E))$. Then,

$$
\sum_{j} \sigma_{j}(t) h^{0}\left(E^{\prime} \otimes L_{j}^{n} \otimes B_{j}\right) P_{E}^{t}(m) \leq \sum_{j} \sigma_{j}(t) h^{0}\left(E^{\prime} \otimes L_{j}^{m} \otimes B_{j}\right) P_{E}^{t}(n) .
$$

Now by (C5') (since $t$ is assumed to be among the $\left\{t_{i}\right\}$ ) we deduce

$$
P_{E}^{t} \sum_{j} \sigma_{j}(t) h^{0}\left(E^{\prime} \otimes L_{j}^{n} \otimes B_{j}\right) \leq P_{E^{\prime}}^{t} P_{E}^{t}(n)
$$

and so taking the leading order term in these polynomials

$$
r_{E}^{t} \sum_{j} \sigma_{j}(t) h^{0}\left(E^{\prime} \otimes L_{j}^{n} \otimes B_{j}\right) \leq r_{E^{\prime}}^{t} P_{E}^{t}(n) .
$$

Thus, by the Le Potier-Simpson Theorem (as discussed in Remark 2.8), this implies $p_{E^{\prime}}^{t} \leq p_{E}^{t}$, which contradicts our choice of $E^{\prime}$.

Lemma 2.15. Suppose an $A$-module $M$ is semistable with respect to $t^{\prime} \in(0,1)$ and not semistable with respect to $t^{\prime \prime} \in(0,1)$. Then there exists a $t$ between $t^{\prime}$ and $t^{\prime \prime}$ and a submodule $M^{\prime}$ of $M$ such that

(1) $M$ is properly semistable with respect to $t$.

(2) $\mu_{t}\left(M^{\prime}\right)=\mu_{t}(M)$.

(3) The function $s \mapsto \mu_{s}\left(M^{\prime}\right)-\mu_{s}(M)$ is not identically zero.

Proof. Assume $t^{\prime}<t^{\prime \prime}$ (the other case being proved in the same way). The set $\mathcal{D}$ of dimension vectors $\underline{e}$ of non-zero submodules $M^{\prime}$ of $M$ is finite. For each such $\underline{e} \in \mathcal{D}$ set

$$
g_{\underline{e}}(s):=\mu_{s}\left(M^{\prime}\right)-\mu_{s}(M) \text { for } s \in\left[t^{\prime}, t^{\prime \prime}\right]
$$

and

$$
\mathcal{D}_{0}:=\left\{\underline{e} \in \mathcal{D}: g_{\underline{e}}(\cdot) \text { is not identically } 0\right\}
$$


As $M$ is not semistable with respect to $t^{\prime \prime}$ the set $\mathcal{D}_{0}$ is non-empty.

Now if $\underline{e} \in \mathcal{D}_{0}$ we have $g_{\underline{e}}\left(t^{\prime}\right) \leq 0$ as $M$ is semistable with respect to $t^{\prime}$. Define

$$
t:=\sup \left\{s \in\left[t^{\prime}, t^{\prime \prime}\right): g_{\underline{e}} \leq 0 \text { on }\left[t^{\prime}, s\right] \text { for all } \underline{e} \in \mathcal{D}_{0}\right\} .
$$

Then clearly $M$ is semistable with respect to $t$, and there must be some $\underline{e} \in \mathcal{D}_{0}$ for which $g_{\underline{e}}(t)=0$. Letting $M^{\prime}$ be a submodule with dimension vector $\underline{e}^{\prime}$ proves the lemma.

Proof of Theorem 2.9. Suppose first $E$ is semistable with respect to some $t \in\left[t^{\prime}, t^{\prime \prime}\right]$. Then by definition it is torsion-free, and it is $(p, \underline{L}, \underline{B})$-regular by choice of $p$. We aim to show that $M:=\operatorname{Hom}(T, E)$ is also semistable with respect to $t$.

To this end, suppose first that $t=t_{i}$ for some $i$. Then, certainly $M$ is semistable by the Preservation of Semistability with respect to these points (cf. Theorem 2.11). Thus, we may assume $t \in\left(t_{i}, t_{i+1}\right)$ for some $i$. Then, $E$ must by also semistable with respect to all $t^{\prime} \in\left[t_{i}, t_{i+1}\right]$, since by construction $t_{i}$ were the walls at which semistability of sheaves of this type may change and our stability segment is assumed to be uniform. In particular this is true at the endpoints, so once again by Theorem 2.11 the module $M$ is semistable with respect to $t_{i}$ and $t_{i+1}$.

Suppose for contradiction $M$ is not semistable with respect to this $t$. By Lemma 2.15 there is some $t^{\prime} \in\left[t_{i}, t_{i+1}\right]$ and a submodule $M^{\prime} \subset M$ such that $M$ is properly semistable with respect to $t^{\prime}, \mu_{t^{\prime}}\left(M^{\prime}\right)=\mu_{t^{\prime}}(M)$, and so $s \mapsto \mu_{s}\left(M^{\prime}\right)-\mu_{s}(M)$ is not identically zero. Note that this function is not linear in general.

Thus, we can apply Lemma 2.13 to deduce there is an $(n, \underline{L}, \underline{B})$-regular subsheaf $E^{\prime} \subset E$ such that $M^{\prime}$ is subordinate to $\operatorname{Hom}\left(T, E^{\prime}\right)$. So

$$
\mu_{t^{\prime}}\left(\operatorname{Hom}\left(T, E^{\prime}\right)\right) \geq \mu_{t^{\prime}}\left(M^{\prime}\right)=\mu_{t^{\prime}}(M)
$$

and hence $\mu_{t^{\prime}}\left(\operatorname{Hom}\left(T, E^{\prime}\right)\right)=\mu_{t^{\prime}}(M)$ by semistability of $M$ with respect to $t^{\prime}$. So $\mu_{t^{\prime}}\left(M^{\prime}\right)=$ $\mu_{t^{\prime}}\left(\operatorname{Hom}\left(T, E^{\prime}\right)\right)$ and thus in fact $M^{\prime}=\operatorname{Hom}\left(T, E^{\prime}\right)$.

Now by Lemma 2.4, since we are assuming that our stability segment is uniform, the function

$$
f_{E^{\prime}}(s)=P_{E^{\prime}}^{s}(n) P_{E}^{s}(m)-P_{E}^{s}(n) P_{E^{\prime}}^{s}(m)
$$

is linear in $s$, and by $(n, \underline{L}, \underline{B})$-regularity of both $E^{\prime}$ and $E$

$$
\begin{aligned}
f_{E^{\prime}}(s) & =P_{E^{\prime}}^{s}(m) P_{E}^{s}(m)\left(\frac{P_{E^{\prime}}^{s}(n)}{P_{E^{\prime}}^{s}(m)}-\frac{P_{E}^{s}(n)}{P_{E}^{s}(m)}\right) \\
& =P_{E^{\prime}}^{s}(m) P_{E}^{s}(m)\left(\mu_{s}\left(\operatorname{Hom}\left(T, E^{\prime}\right)\right)-\mu_{s}(M)\right) .
\end{aligned}
$$

But this is absurd, since by the above $f_{E^{\prime}}(\cdot)$ is linear and not identically zero, $f_{E^{\prime}}\left(t^{\prime}\right)=0$, but $f_{E^{\prime}}\left(t_{i}\right) \leq 0$ and $f_{E^{\prime}}\left(t_{i+1}\right) \leq 0$, as $\operatorname{Hom}(T, E)$ is semistable at $t_{i}$ and $t_{i+1}$. Thus, we have shown that $M$ is semistable with respect to $t$, as required.

For the converse, suppose $M$ is semistable with respect to some $t \in\left[t_{i}, t_{i+1}\right]$ and we wish to show that $E$ is semistable with respect to $t$.

As above, by Theorem 2.11 we are done if $t$ is in the set $\left\{t_{i}\right\}$, so we may assume $t \in\left(t_{i}, t_{i+1}\right)$. We suppose for contradiction $E$ is not semistable with respect to $t$. Then, there exists a saturated $E^{\prime} \subset E$ with $p_{E^{\prime}}^{t}>p_{E}^{t}$. So certainly $\hat{\mu}_{E^{\prime}}^{t} \geq \hat{\mu}_{E}^{t}$ and by $\left(\mathrm{C} 2^{\prime}\right) E^{\prime}$ is then $(n, \underline{L}, \underline{B})$ regular. Now clearly we must either have $p_{E^{\prime}}^{t_{i}}>p_{E}^{t_{i}}$ or $p_{E^{\prime}}^{t_{i+1}}>p_{E}^{t_{i}+1}$ by linearity of the $\sigma_{j}$ and by uniformity of $(\sigma(t))_{t \in[0,1]}$. From Lemma 2.14 this implies either $\mu_{t_{i}}\left(\operatorname{Hom}\left(T, E^{\prime}\right)\right)>\mu_{t_{i}}(M)$ or $\mu_{t_{i+1}}\left(\operatorname{Hom}\left(T, E^{\prime}\right)\right)>\mu_{t_{i+1}}(M)$. Thus, with $f_{E^{\prime}}$ as in (2.13) above we deduce from (2.15) that $f_{E^{\prime}}\left(t_{i}\right)>0$ or $f_{E^{\prime}}\left(t_{i+1}\right)>0$. In particular, we conclude $f_{E^{\prime}}$ is not identically zero. But 
$M$ was assumed to be semistable with respect to $t$, so certainly $f_{E^{\prime}}(t) \leq 0$, which by our choice of $\left\{t_{i}\right\}$ implies $t=t_{i}$ or $t=t_{i+1}$ which is absurd. Thus, $E$ is semistable as claimed.

Finally, we turn to the statement about $S$-equivalence. By Theorem 2.11, the statement we want holds at any point in $\left\{t_{i}\right\}$, so our interest lies in the open interval $\left(t_{i}, t_{i+1}\right)$. Again by construction, the $S$-equivalence class of any semistable $E$ taken with respect to any point within $\left(t_{i}, t_{i+1}\right)$ is independent of that point. We claim that the same is true for the semistable module $M=\operatorname{Hom}(T, E)$.

To see this, suppose that $M$ is semistable with respect to some point $t \in\left(t_{i}, t_{i+1}\right)$ and that $M^{\prime} \subset M$ is destabilising with respect to $t$.

By Lemma 2.13 we may find a subsheaf $E^{\prime}$ of $E$ such that $M^{\prime}$ is subordinate to $\operatorname{Hom}\left(T, E^{\prime}\right)$ and thus $\mu_{t}\left(M^{\prime}\right) \leq \mu_{t}\left(\operatorname{Hom}\left(T, E^{\prime}\right)\right)$ [GRT14, Lemma 8.11]. Hence, we may suppose that $M^{\prime}=\operatorname{Hom}\left(T, E^{\prime}\right)$ for some $E^{\prime} \subset E$ that is $(n, \underline{L}, \underline{B})$-regular. So the function $f_{E^{\prime}}(\cdot)$ is linear. Thus, we have $f_{E^{\prime}}(t)=0$ but $f_{E^{\prime}}(\cdot) \leq 0$ over all of $\left(t_{i}, t_{i+1}\right)$ by semistability of $M$. Therefore, $f_{E^{\prime}} \equiv 0$, and so $M^{\prime}$ is a destabilising submodule of $M$ with respect to any point in $\left(t_{i}, t_{i+1}\right)$.

Now, by Theorem 2.11 applied to the chosen points $t_{i}^{\prime} \in\left(t_{i}, t_{i+1}\right)$ we have $\operatorname{Hom}(T, \operatorname{gr}(E))$ is isomorphic to $\operatorname{gr}(\operatorname{Hom}(T, E))$. Hence, this must also hold for all points in $\left(t_{i}, t_{i+1}\right)$, as the isomorphism class of both sides are unchanged within this interval, completing the proof.

2.3. Uniform Variation (Proof of Theorem 2.6). The proof of Theorem 2.6, which identifies the intermediate spaces, is essentially the same as that for a finite set of stability parameters as in [GRT14, Section 10]. There, we considered the union $Y=\bigcup_{\sigma \in \Sigma^{\prime}} Q^{[\sigma-s s]} \subset R$ as $\sigma$ varies in a finite set $\Sigma^{\prime}$ of stability parameters, together with its closure $Z=\bar{Y}$. This is now replaced by $Y=\bigcup_{t \in\left[t^{\prime}, t^{\prime \prime}\right]} Q^{[\sigma(t)-s s]}$, which is still a finite union as $\sigma(t)_{t \in\left[t^{\prime}, t^{\prime \prime}\right]}$ admits a finite chamber structure, and its closure $Z=\bar{Y}$. By the Uniform Comparison of Semistability, Theorem 2.9, the "master space" statement [GRT14, Theorem 10.1]

$$
Z^{\sigma(t)}:=R^{\sigma(t)-s s} \cap Z=Q^{[\sigma(t)-s s]}
$$

now holds for all $t \in\left[t^{\prime}, t^{\prime \prime}\right]$. In [GRT14, Corollary 10.2] we identified a cone $\mathcal{C}_{G}(Z)$ together with a chamber decomposition reflecting the change of sets of semistable points. So, as $\sigma(t)$ varies with $t$, we have a path $\theta_{\sigma(t)}$ of characters which pass through the various chambers, that precisely witness the Thaddeus-flips that occur. By the uniform statements in the previous section we know that each of these is a moduli space of sheaves, precisely as in proof of [GRT14, Corollary 10.2].

\section{Chamber structure for Gieseker-Stability}

Our goal here is to exhibit a chamber structure on the ample cone of a projective manifold that witnesses the change in Gieseker-stability as the polarisation varies. We will use the results and notation setup here to define later a precise notion of a "general" polarisation to which our main variation result in the next section applies.

Assume that $X$ is smooth of dimension $d$ over an algebraically closed field of characteristic zero, and let $\tau \in B(X)_{\mathbb{Q}}$. We recall the slope of a torsion-free sheaf $E$ on $X$, as in [GRT14, Definition 3.1], with respect to $L \in \operatorname{Amp}(X)_{\mathbb{R}}$, or a curve class $\gamma \in N_{1}(X)_{\mathbb{R}}$ is

$$
\mu^{L}(E)=\frac{\int_{X} c_{1}(E) c_{1}(L)^{d-1}}{\operatorname{rank}(E)} \text { and } \mu_{\gamma}(E)=\frac{\int_{X} c_{1}(E) \cdot \gamma}{\operatorname{rank}(E)} .
$$

We also recall $\operatorname{Pos}(X)_{\mathbb{R}}:=\left\{\gamma \in N_{1}(X)_{\mathbb{R}}: \gamma=D^{d-1}\right.$ for some $\left.D \in \operatorname{Amp}(X)_{\mathbb{R}}\right\}$ and that the $\operatorname{map} p: \operatorname{Amp}(X)_{\mathbb{R}} \rightarrow \operatorname{Pos}(X)_{\mathbb{R}}$ given by $p(x)=x^{d-1}$ is a homeomorphism [GT13, Prop. 6.5]. 
Observe that by definition for any torsion-free sheaf $E$ and any $L \in \operatorname{Amp}(X)_{\mathbb{R}}$ we have $\mu^{L}(E)=\mu_{p(L)}(E)$.

Now, suppose that $K \subset \operatorname{Amp}(X)_{\mathbb{R}}$ is open and relatively compact and let

$$
\text { Cone }^{*}(K):=\left\{\lambda L \in \operatorname{Amp}(X)_{\mathbb{R}} \mid L \in K \text { and } \lambda \in \mathbb{R}_{>0}\right\}
$$

be the cone over $K$ with the origin removed.

Lemma 3.1. Let $\mathcal{S}^{\prime}$ be a bounded family of torsion-free sheaves of topological type $\tau$. Then, the set

$$
\mathcal{S}:=\left\{\begin{array}{l|l}
F & \begin{array}{l}
F \text { is a saturated subsheaf of some } E \in \mathcal{S}^{\prime} \text { and } \\
\mu_{\gamma}(F) \geq \mu_{\gamma}(E) \text { for some } \gamma \text { in the convex hull of } p\left(\mathrm{Cone}^{*}(K)\right)
\end{array}
\end{array}\right\}
$$

is bounded.

Proof. Observe the inequality $\mu_{\gamma}(F) \geq \mu_{\gamma}(E)$ is invariant under replacing $\gamma$ by a positive multiple. So, as Convexhull $\left(p\left(\right.\right.$ Cone $\left.^{*}(K)\right) \subset$ Cone $^{*}(\operatorname{Convexhull}(p(K))$, it is sufficient to prove the theorem with Cone* $(K)$ replaced with $K$.

Fix $\tilde{\gamma}$ in the convex hull of $p(\bar{K})$. Then, we can find $\gamma_{1}, \ldots, \gamma_{m} \in \operatorname{Pos}(X)_{\mathbb{R}}$ so that $\gamma_{i}=p\left(A_{i}\right)$ for some $A_{i} \in \operatorname{Amp}(X)_{\mathbb{Q}}$ and so $\tilde{\gamma}$ lies in the interior of the convex hull $H$ of $\gamma_{1}, \ldots, \gamma_{m}$. We claim that the set

$\mathcal{T}_{H}:=\left\{F: F\right.$ is a saturated subsheaf of an $E \in \mathcal{S}^{\prime}$ and $\mu_{\gamma}(F) \geq \mu_{\gamma}(E)$ for some $\left.\gamma \in H\right\}$ is bounded. To prove this, suppose $\gamma$ is a convex combination of $\gamma_{1}, \ldots, \gamma_{m}$. If $\mu_{\gamma}(F) \geq \mu_{\gamma}(E)$, then by convexity $\mu_{\gamma_{i}}(F) \geq \mu_{\gamma_{i}}(E)$ for some $i$, which says precisely $\mu^{A_{i}}(F) \geq \mu^{A_{i}}(E)$. Thus, $\mathcal{T}_{H}$ is contained in a finite union of sets that are each bounded by Grothendieck's Lemma, [HL10, Lem. 1.7.9] or [Gro95, Théorème 2.2], proving the claim.

Now, the convex hull of the compact set $p(\bar{K})$ is also compact (a simple corollary of Carathéodory's theorem) and so can be covered with a finite union of sets of form $H$. Hence, we deduce that $\mathcal{S}$ is contained in a finite union of sets of the form $\mathcal{T}_{H}$, and thus is also bounded as required.

We apply the preceding discussion as follows. Define

$$
\mathcal{S}_{K}^{\prime}:=\left\{E \mid \begin{array}{l}
E \text { is torsion-free of topological type } \tau \text { that is } \\
\text { slope semistable with respect to some } \gamma \in p\left(\text { Cone }^{*}(K)\right)
\end{array}\right\} .
$$

Then, as slope semistability is invariant under scaling $\gamma$ by a positive multiple, this set is unchanged if Cone* $(K)$ is replaced with $K$. Thus, $\mathcal{S}_{K}^{\prime}$ is bounded by [GRT14, Theorem 6.8]. Consequently, by Lemma 3.1 the set

$$
\mathcal{S}_{K}:=\left\{\begin{array}{l|l}
F \mid \begin{array}{l}
F \text { is a saturated subsheaf of some } E \in \mathcal{S}_{K}^{\prime} \text { and } \\
\mu^{L}(F) \geq \mu^{L}(E) \text { for some } L \in \operatorname{Cone}^{*}(K)
\end{array}
\end{array}\right\}
$$

is also bounded. Now, for each $F \in \mathcal{S}_{K}$ write the corresponding difference of reduced Hilbertpolynomials as

$$
p_{F}^{L}(k)-p_{E}^{L}(k)=\frac{1}{\operatorname{vol}(L)} \sum_{i=1}^{d} \beta_{F, i}^{L} \frac{k^{d-i}}{(d-i) !},
$$

where $E$ is any sheaf of topological type $\tau$ and $\operatorname{vol}(L):=\int_{X} c_{1}(L)^{d}$. Note that here we allow $L$ to be real, so these reduced Hilbert polynomials should be defined using the Riemann-Roch 
theorem (as discussed in [GRT14, Definition 11.1], i.e.,

$$
p_{F}^{L}(k):=\frac{1}{\operatorname{rank}(F) \operatorname{vol}(L)} \int_{X} e^{k c_{1}(L)} \operatorname{ch}(F) \operatorname{Todd}(X) .
$$

Hence, $L \mapsto \beta_{F, i}^{L}$ is a polynomial function on $\operatorname{Amp}(X)_{\mathbb{R}}$ of degree $d-i$ (so in particular $\beta_{F, d}^{L}$ is independent of $L$ ).

Moreover, for $i=1, \ldots, d-1$ and $F \in \mathcal{S}_{K}$ we set

$$
\tilde{W}_{F, i}=\left\{L \in \operatorname{Amp}(X)_{\mathbb{R}} \mid \beta_{F, i}^{L}=0\right\}
$$

which we shall refer to as a wall. So, each such wall is either empty, all of $\operatorname{Amp}(X)_{\mathbb{R}}$, or a non-trivial real algebraic variety in $\operatorname{Amp}(X)_{\mathbb{R}}$.

Definition 3.2. We let $\tilde{\mathcal{W}}_{K}$ be the set of walls $\tilde{W}_{F, i}$ for $F \in \mathcal{S}_{K}$ and $i=1, \ldots, d-1$ such that $\tilde{W}_{F, i}$ is neither empty nor all of $\operatorname{Amp}(X)_{\mathbb{R}}$.

Observe that $\tilde{W}_{F, i}$ depends only on the topological type of $F$, and by boundedness of $\mathcal{S}_{K}$ there are only finitely many such types. Hence, the set $\tilde{\mathcal{W}}_{K}$ consists of a finite number of (non-trivial) walls that divide $\operatorname{Cone}^{*}(K)$ into a number of chambers (see [GRT14, Definition 4.1] for the precise definition of a chamber).

Proposition 3.3 (Existence of chamber structure for Gieseker-stability). The collection $\tilde{\mathcal{W}}_{K}$ of walls gives a chamber structure on $\operatorname{Cone}^{*}(K)$ that witnesses the change in Gieseker-stability as $L$ varies within $\operatorname{Cone}^{*}(K)$. More precisely, if $L^{\prime}, L^{\prime \prime} \in \operatorname{Cone}^{*}(K)$ lie in the same chamber, then for any sheaf $E$ of topological type $\tau$ it holds that

(1) E is Gieseker-(semi)stable with respect to $L^{\prime}$ if and only if it is Gieseker-(semi)stable with respect to $L^{\prime \prime}$.

(2) If $E$ is Gieseker-(semi)stable with respect to $L^{\prime}$ and $L^{\prime \prime}$ then the $S$-equivalence class of $E$ is the same taken with respect to either $L^{\prime}$ or $L^{\prime \prime}$.

Proof. The proof of this is essentially the same as that of [GRT14, Proposition 4.2] and can be found in [GRT15, Proposition 3.3].

\section{Mumford-Thaddeus PRINCIPLE FOR GENERAL LINE BUNDLES}

In [GRT14] we proved that any two moduli spaces of Gieseker-semistable sheaves on a smooth projective threefold are connected by a finite number of Thaddeus-flips. In this section, we will extend this structure result to base manifolds of arbitrary dimensions. However, for this we have to restrict to general classes in $N_{1}(X)_{\mathbb{R}}$, which we now make precise.

Definition 4.1. We say that $L \in \operatorname{Amp}(X)_{\mathbb{R}}$ is general if there exists an open relatively compact $K \subset \operatorname{Amp}(X)_{\mathbb{R}}$ with $L \in \operatorname{Cone}^{*}(K)$ such that $L$ does not lie on any of the walls in $\tilde{\mathcal{W}}_{K}$.

As is clear from the definition, choosing such a $K$, the set of points in Cone* $(K)$ that are general are dense in $\operatorname{Cone}^{*}(K)$. In fact they consist of the complement of a finite number of algebraic varieties, from which one sees that in fact the points $\operatorname{Amp}(X)_{\mathbb{Q}}$ that are general are dense in $\operatorname{Amp}(X)_{\mathbb{R}}$. Observe finally that if $L$ is general then so is $L^{r}$ for all $r \in \mathbb{R}_{>0}$.

Now let $K \subset \operatorname{Amp}(X)_{\mathbb{R}}$ be open and relatively compact, and recall the sets $\mathcal{S}_{K}^{\prime}$ and $\mathcal{S}_{K}$ were defined in (3.1) and (3.2), respectively. 
Lemma 4.2. Let $\sigma^{\prime}=\left(\underline{L}^{\prime}, \sigma_{1}^{\prime}, \ldots, \sigma_{j_{0}}^{\prime}\right)$ and $\sigma^{\prime \prime}=\left(\underline{L}^{\prime \prime}, \sigma_{1}^{\prime \prime}, \ldots, \sigma_{j_{0}}^{\prime \prime}\right)$ be stability parameters. Set

$$
\gamma^{\prime}:=\sum_{j} \sigma_{j}^{\prime} c_{1}\left(L_{j}^{\prime}\right)^{d-1} \text { and } \gamma^{\prime \prime}:=\sum_{j} \sigma_{j}^{\prime \prime} c_{1}\left(L_{j}^{\prime \prime}\right)^{d-1}
$$

and suppose that $\gamma^{\prime}$ and $\gamma^{\prime \prime}$ both lie in $p\left(\operatorname{Cone}^{*}(K)\right)$. Suppose in addition that for any torsionfree sheaf $E$ of topological type $\tau$ and any saturated $F \subset E$ with $F \in \mathcal{S}_{K}$ it holds that

$$
p_{F}^{\sigma^{\prime}}(\leq) p_{E}^{\sigma^{\prime}} \text { if and only if } p_{F}^{\sigma^{\prime \prime}}(\leq) p_{E}^{\sigma^{\prime \prime}}
$$

Then, such a sheaf $E$ is (semi)stable with respect to $\sigma^{\prime}$ if and only if it is (semi)stable with respect to $\sigma^{\prime \prime}$.

Proof. Suppose $E$ is semistable with respect to $\sigma^{\prime}$. Then, it is slope semistable with respect to $\gamma^{\prime}$ which says $E \in \mathcal{S}_{K}^{\prime}$. To test for (semi)stability of $E$ with respect to $\sigma^{\prime \prime}$ it is sufficient to consider only saturated subsheaves $F$ of $E$. If $\mu_{\gamma^{\prime \prime}}(F)<\mu_{\gamma^{\prime \prime}}(E)$, then clearly $F$ does not destabilise $E$ with respect to $\sigma^{\prime \prime}$ (cf. [GRT14, Example 2.6], or Remark 1.3). Otherwise $\mu_{\gamma^{\prime \prime}}(F) \geq \mu_{\gamma^{\prime \prime}}(E)$ and so $F \in \mathcal{S}_{K}$ and we are done.

Proposition 4.3. Let $L^{\prime}, L^{\prime \prime} \in \operatorname{Amp}(X)$ and suppose that $L^{\prime}$ is general. Consider the family of stability parameters

$$
\sigma(t):=\left(L^{\prime}, L^{\prime \prime}, \frac{1-t}{\operatorname{vol}\left(L^{\prime}\right)}, \frac{t}{\operatorname{vol}\left(L^{\prime \prime}\right)}\right) \text { for } t \in[0,1] .
$$

Then, for all $t>0$ sufficiently small

(1) Any sheaf of topological type $\tau$ is Gieseker-(semi)stable with respect to $L^{\prime}$ if and only if it is $\sigma(t)$-(semi) stable

(2) Suppose $E$ and $E^{\prime}$ are sheaves of topological type $\tau$ that are Gieseker-semistable with respect to $L^{\prime}$. Then, $E$ and $E^{\prime}$ are $S$-equivalent in terms of Gieseker-stability defined using $L^{\prime}$ if and only if they are $S$-equivalent in terms of multi-Gieseker-stability defined using $\sigma(t)$.

Thus, for $t>0$ sufficiently small the stability parameter $\sigma(t)$ is bounded, and $\mathcal{M}_{L^{\prime}}=\mathcal{M}_{\sigma(t)}$.

Proof. As $L^{\prime}$ is general, there exists an open relatively compact $K \subset \operatorname{Amp}(X)_{\mathbb{R}}$ with $L^{\prime} \in$ Cone* $(K)$ and $L^{\prime}$ not lying on any wall in $\tilde{\mathcal{W}}_{K}$.

Let $E$ be a sheaf of topological type $\tau$. If $F \subset E$ is saturated with $F \in \mathcal{S}_{K}$ we write the difference of reduced multi-Hilbert-polynomials as

$$
p_{F}^{\sigma(t)}(k)-p_{E}^{\sigma(t)}(k)=\sum_{i=1}^{d} h_{F, i}(t) \frac{k^{d-i}}{(d-i) !} .
$$

Clearly $h_{F, i}$ depends only on the topological type of $F$. Moreover, since for any sheaf $F$ we have $p_{F}^{\sigma(0)}(k)=\frac{1}{\operatorname{rank}(F) \operatorname{vol}\left(L^{\prime}\right)} \chi\left(F \otimes L^{\prime k}\right)=p_{F}^{L^{\prime}}(k)$, it holds that $h_{F, i}(0)$ is a positive multiple of $\beta_{F, i}^{L^{\prime}}$ (as defined in (3.3)), and similarly $h_{F, i}(1)$ and $\beta_{F, i}^{L^{\prime \prime}}$. In fact, using the Riemann-Roch theorem (cf. [GRT14, Example 2.6] or Remark 1.3) one checks easily that $h_{F, i}:[0,1] \rightarrow \mathbb{R}$ is linear.

We claim that for each $i=1, \ldots, d$ and $F \in \mathcal{S}_{K}$ either $h_{F, i}(t)=0$ for all $t \in[0,1]$, or $h_{F, i}(0) \neq 0$. This is clear when $i=d$ for then $h_{F, i}$ is independent of $t$, so assume $1 \leq i \leq d-1$ and $h_{F, i}(0)=0$. So $\beta_{F, i}^{L^{\prime}}=0$, so $L^{\prime}$ lies on the wall $\tilde{W}_{F, i}$. So as $L^{\prime}$ is general this implies that $\tilde{W}_{F, i}$ is trivial, i.e. is the whole of $\operatorname{Amp}(X)_{\mathbb{R}}$. In particular $L^{\prime \prime} \in \tilde{W}_{F, i}$, and thus $h_{F, i}(1)=0$ as well, and so by linearity $h_{F, i}(t)=0$ for all $t \in[0,1]$, thus proving the claim. 
Now, as there are only a finite number of topological types among the sheaves in $\mathcal{S}_{K}$, for all $t_{0}>0$ sufficiently small the following holds: for all $F \in \mathcal{S}_{K}$ and all $i=1, \ldots, d$ either

$$
h_{F, i} \text { has no roots in }\left[0, t_{0}\right] \text { or } h_{F, i}(t)=0 \text { for all } t \in[0,1] \text {. }
$$

We now prove (1) by showing that $E$ is (semi)stable with respect to $\sigma(0)$ (which is equivalent to being Gieseker-(semi)stable with respect to $L^{\prime}$ ) if and only if it is (semi)stable with respect to $\sigma\left(t_{0}\right)$. To this end, let

$$
\gamma_{t}=\frac{1-t}{\operatorname{vol}\left(L^{\prime}\right)} c_{1}\left(L^{\prime}\right)^{d-1}+\frac{t}{\operatorname{vol}\left(L^{\prime \prime}\right)} c_{1}\left(L^{\prime \prime}\right)^{d-1} \in N_{1}(X)_{\mathbb{R}} .
$$

As $L^{\prime} \in$ Cone $^{*}(K)$, we have $\gamma_{0} \in p\left(\right.$ Cone $\left.^{*}(K)\right)$ and by continuity, by shrinking $t_{0}$ if necessary, we have $\gamma_{t_{0}} \in p\left(\right.$ Cone $\left.^{*}(K)\right)$ as well. So we are in a position to apply Lemma 4.2 , so it is sufficient to prove that for all saturated $F \subset E$ with $F \in \mathcal{S}_{K}$ we have

$$
p_{F}^{\sigma(0)}(\leq) p_{E}^{\sigma(0)} \text { if and only if } p_{F}^{\sigma\left(t_{0}\right)}(\leq) p_{E}^{\sigma\left(t_{0}\right)} .
$$

But precisely from the ordering of polynomials (which is the same as the lexicographic ordering on its coefficients) this follows immediately from (4.2), proving statement (1) in the theorem. The statement (2) about $S$-equivalence then follows from this.

Finally, as is well known, the set of sheaves of a given topological type that are semistable with respect to $L^{\prime}$ is bounded, by [HL10, 3.3.7], or [GRT14, Theorem 6.11]. Thus, for $t>0$ sufficiently small, $\sigma(t)$ is also bounded, and the final statement follows.

Theorem 4.4 (Mumford-Thaddeus principle for Gieseker moduli spaces). Let $X$ be a projective manifold of dimension $d$ over an algebraically closed field of characteristic zero, let $\tau \in B(X)_{\mathbb{Q}}$, and let $L^{\prime}, L^{\prime \prime} \in \operatorname{Amp}(X)_{\mathbb{Q}}$ be general. Then, the moduli spaces $\mathcal{M}_{L^{\prime}}$ and $\mathcal{M}_{L^{\prime \prime}}$ of torsion-free sheaves of topological type $\tau$ that are Gieseker-semistable with respect to $L^{\prime}$ and $L^{\prime \prime}$, respectively, are related by a finite number of Thaddeus-flips.

Proof of Theorem 4.4. Consider the segment of stability parameters given by

$$
\sigma(t)=\left(L^{\prime}, L^{\prime \prime}, \frac{1-t}{\operatorname{vol}\left(L^{\prime}\right)}, \frac{t}{\operatorname{vol}\left(L^{\prime \prime}\right)}\right) \text { for } t \in[0,1] .
$$

As $L^{\prime}$ and $L^{\prime \prime}$ are general, two applications of Proposition 4.3 imply that for $t>0$ sufficiently small $\mathcal{M}_{L^{\prime}}=\mathcal{M}_{\sigma(t)}$ and $\mathcal{M}_{L^{\prime \prime}}=\mathcal{M}_{\sigma(1-t)}$. Moreover, as $t \in(0,1)$, both $\sigma(t)$ and $\sigma(1-t)$ are clearly positive stability parameters, and so [GRT14, Theorem 10.1] applies to give the result.

\section{Gieseker-Stability With Respect to ReAl Ample Classes}

We digress to revisit the moduli spaces $\mathcal{M}_{\omega}$ of Gieseker-semistable sheaves taken with respect to a real class $\omega \in \operatorname{Amp}(X)_{\mathbb{R}}$. Suppose we again fix an open, relatively compact $K \subset \operatorname{Amp}(X)_{\mathbb{R}}$ and assume that $\omega \in \operatorname{Cone}^{*}(K)$. If $\omega$ is general, so does not lie on any of the walls in $\tilde{\mathcal{W}}_{K}$, then we can perturb it slightly to find a rational $L \in \operatorname{Amp}(X)_{\mathbb{Q}}$ in the same chamber. Thus, Gieseker-stability with respect to $\omega$ is the same as that for $L$, and so the moduli spaces $\mathcal{M}_{\omega}=\mathcal{M}_{L}$ agree, and so in particular $\mathcal{M}_{\omega}$ is projective.

We now show that we can still get such a projective moduli space if $\omega$ is allowed to lie on walls of the form $\tilde{W}_{1, F}$ for $F \in \mathcal{S}_{K}$, but not on any walls of the form $\tilde{W}_{i, F}$ for $F \in \mathcal{S}_{K}$ and $i \geq 2$. 
Theorem 5.1 (Projective moduli spaces for $\omega$-semistable sheaves). Let $X$ be a smooth projective manifold of dimension $d$ and $\tau \in B(X)_{\mathbb{Q}}$. Suppose that $K \subset \operatorname{Amp}(X)_{\mathbb{R}}$ is open and relatively compact and that $\omega \in \operatorname{Cone}^{*}(K)$ does not lie on any of the walls $\tilde{W}_{i, F}$ for $i \geq 2$ and $F \in \mathcal{S}_{K}$.

Then, there exists a projective moduli space $\mathcal{M}_{\omega}$ of torsion-free sheaves of topological type $\tau$ that are Gieseker-semistable with respect to $\omega$. This moduli space contains an open set consisting of points representing isomorphism classes of stable sheaves, while points on the boundary correspond to $S$-equivalence classes of strictly semistable sheaves.

Proof. Clearly $\omega^{n-1} \in \operatorname{Pos}(X)_{\mathbb{R}}$. As the map $p: \operatorname{Amp}(X)_{\mathbb{R}} \rightarrow \operatorname{Pos}(X)_{\mathbb{R}}$ is a homeomorphism we can find $L_{1}, \ldots, L_{j_{0}} \in \operatorname{Amp}(X)_{\mathbb{Q}}$ arbitrarily close to $\omega$, so that $\omega^{n-1}$ is a convex combination of $p\left(L_{1}\right), \ldots, p\left(L_{j_{0}}\right)$, say $\omega^{n-1}=\sum_{j} \sigma_{j} L_{j}^{d-1}$. By rescaling all the $L_{j}$ simultaneously we may assume all of them to be integral. We then set $\sigma=\left(L_{1}, \ldots, L_{j_{0}}, \sigma_{1}, \ldots, \sigma_{j_{0}}\right)$.

As $\omega$ does not lie on any of the walls $\tilde{W}_{i, F}$ for $i \geq 2$, we can take such $L_{j}$ close enough to $\omega$ to ensure that $\beta_{i, F}^{L}$ has the same sign as $\beta_{i, F}^{\omega}$ for all $i \geq 2$ and $F \in \mathcal{S}_{K}$. Using convexity, one checks that this implies that $p_{F}^{\omega}(\leq) p_{F}^{\omega}$ if and only if $p_{F}^{\sigma}(\leq) p_{E}^{\sigma}$. So $E$ is Gieseker-(semi)stable with respect to $\omega$ if and only if it is (semi)stable with respect to $\sigma$. Now as the wall structure for multi-Gieseker stability is rational linear [GRT14, Proposition 4.2] we can perturb the $\sigma_{j}$ to be rational without changing stability, and thus $\mathcal{M}_{\omega}=\mathcal{M}_{\sigma}$.

\section{Construction of Uniform Stability Segments}

In this section we discuss a method for construction uniform stability segments on surfaces and threefolds. The upshot on surfaces will be that we recover, in a natural way, the main result of Matsuki-Wentworth [MW97] (see Section 6.3). Thus we shall focus instead on the case of threefolds with the aim of proving the following:

Theorem 6.1 (Identification of intermediate spaces). Let $X$ be a smooth projective manifold of dimension 3. Suppose $L_{0}$ and $L_{1}$ are ample line bundles "separated by a single wall of the first kind". Then, the moduli spaces $\mathcal{M}_{L_{0}}$ and $\mathcal{M}_{L_{1}}$ of Gieseker-semistable torsion-free sheaves of topological type $\tau$ are related by a finite number of Thaddeus-flips through spaces of the form $\mathcal{M}_{\sigma}$ for some bounded stability parameter $\sigma$.

The definition of what it means to be "separated by a single wall of the first kind" will be given below (Definition 6.4).

6.1. Notation and setup for the proof of Theorem 6.1. In order to shorten notation, from here on, a given stability segment $(\sigma(t))_{t \in[0,1]}$ will be written as $\sigma(\cdot)$. As we are interested in the moduli spaces, rather than the precise stability parameters, the following equivalence relations are convenient:

Definition 6.2. Let $\sigma$ and $\tilde{\sigma}$ be bounded stability parameters.

(1) We write $\sigma \equiv \tilde{\sigma}$ if any sheaf of topological type $\tau$ is (semi)stable with respect to $\sigma$ if and only if it is (semi)stable with respect to $\tilde{\sigma}$.

(2) We write $\mathcal{M}_{\sigma^{\leftarrow-\rightarrow}} \mathcal{M}_{\tilde{\sigma}}$ if the moduli spaces $\mathcal{M}_{\sigma}$ and $\mathcal{M}_{\tilde{\sigma}}$ are related by a finite number of Thaddeus-flips through spaces of the form $\mathcal{M}_{\sigma_{i}}$ for some bounded stability parameters $\sigma_{i}$. 
6.1.1. Riemann-Roch. To simplify various Riemann-Roch calculations, we shall use the following notation adapted from Schmitt [Sch00, Sect. 2.1]. Let $X$ be a smooth manifold of dimension $d$ and $L$ be an ample line bundle on $X$. For a torsion-free sheaf $E$ define

$$
\operatorname{Hilb}_{i}(E):=(\operatorname{ch}(E) \operatorname{Todd}(X))_{i} \quad \text { and } \quad \operatorname{hilb}_{i}(E):=\frac{\operatorname{Hilb}_{i}(E)}{\operatorname{rank}(E)},
$$

where the subscript $i$ denotes the part of this product in $H^{2 i}(X, \mathbb{Q})$. Also, given a proper subsheaf $F \subset E$ we set

$$
\operatorname{hilb}_{i}(F, E):=\operatorname{hilb}_{i}(F)-\operatorname{hilb}_{i}(E) .
$$

For top degree forms we shall omit the integration over $X$ when it is clear from context, so for example

$$
\operatorname{hilb}_{i}(E) c_{1}(L)^{d-i}:=\int_{X} \operatorname{hilb}_{i}(E) c_{1}(L)^{d-i}=\frac{1}{\operatorname{rank}(E)} \int_{X} \operatorname{Hilb}_{i}(E) c_{1}(L)^{d-i} .
$$

Now, for a polynomial of the form

$$
p(k)=p_{1} \frac{k^{d-1}}{(d-1) !}+p_{2} \frac{k^{d-2}}{(d-2) !}+\cdots+p_{d},
$$

we write

$$
p=\left\langle\left\langle p_{1}\left\|p_{2}\right\| \cdots \| p_{d}\right\rangle\right\rangle
$$

for the vector of coefficients. Thus, the ordering on such polynomials $p$ is the lexicographic order on the vector $\left\langle\left\langle p_{1} \| \cdots|| p_{d}\right\rangle\right\rangle$. So, by Riemann-Roch, the reduced Hilbert polynomial of a torsion-free sheaf $E$ with respect to $L$ is $p_{E}^{L}=\frac{k^{d}}{d !}+p(k)$, where

$$
p=\frac{1}{\operatorname{vol}(L)}\left\langle\left\langle\operatorname{hilb}_{1}(E) c_{1}(L)^{d-1}\left\|\operatorname{hilb}_{2}(E) c_{1}(L)^{d-2}\right\| \ldots \| \operatorname{hilb}_{d}(E)\right\rangle\right\rangle .
$$

Finally if $\sigma$ is a stability parameter, then for any proper subsheaf $F \subset E$ we let

$$
p_{F \subset E}^{\sigma}:=p_{F}^{\sigma}-p_{E}^{\sigma} .
$$

6.1.2. Gieseker Walls. We now define what we mean for two line bundles to be separated by a single wall of the first kind. For simplicity here we assume $X$ is smooth of dimension 3 (but a similar story holds in any dimension). Fix a relatively compact open and connected $K \subset \operatorname{Amp}(X)_{\mathbb{R}}$. As defined in Section 3 this gives rise to a collection $\tilde{\mathcal{W}}_{K}$ of non-trivial walls that witnesses the change in Gieseker-stability. For convenience we recall the construction. Let $\operatorname{Cone}^{*}(K)=\left\{\lambda L: L \in K, \lambda \in \mathbb{R}_{>0}\right\}$,

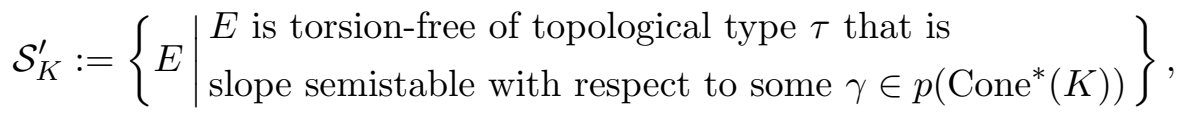

and

$$
\mathcal{S}_{K}:=\left\{\begin{array}{l|l}
F \mid \begin{array}{l}
F \text { is a saturated subsheaf of some } E \in \mathcal{S}_{K}^{\prime} \text { and } \\
\mu^{L}(F) \geq \mu^{L}(E) \text { for some } L \in \operatorname{Cone}^{*}(K)
\end{array}
\end{array}\right\},
$$

which are both bounded. For each $F \in \mathcal{S}_{K}$ write the difference of reduced Hilbert-polynomials as

$$
p_{F}^{L}(k)-p_{E}^{L}(k)=\frac{1}{\operatorname{vol}(L)} \sum_{i=1}^{3} \beta_{F, i}^{L} \frac{k^{d-i}}{(d-i) !}
$$

where $E$ is any sheaf of topological type $\tau$, and for $i=1,2$ let

$$
\tilde{W}_{F, i}=\left\{L \in \operatorname{Amp}(X)_{\mathbb{R}} \mid \beta_{F, i}^{L}=0\right\} .
$$


Definition 6.3. We call the $\tilde{W}_{F, 1}$ walls of the first kind and the $\tilde{W}_{F, 2}$ walls of the second kind. We let $\tilde{\mathcal{W}}_{K}$ be the finite set of walls $\tilde{W}_{F, i}$ for $F \in \mathcal{S}_{K}$ and $i=1,2$ such that $\tilde{W}_{F, i}$ is neither empty nor all of $\operatorname{Amp}(X)_{\mathbb{R}}$.

Definition 6.4. Let $K \subset \operatorname{Amp}(X)_{\mathbb{R}}$ be open and relatively compact. We say that $L_{0}, L_{1} \in$ $\operatorname{Cone}^{*}(K) \cap \operatorname{Amp}(X)_{\mathbb{Q}}$ are separated by a single wall the first kind if

(1) $L_{0}$ and $L_{1}$ do not lie on any of the walls in $\tilde{\mathcal{W}}_{K}$

(2) The straight line segment between $L_{0}$ and $L_{1}$ is contained in Cone* $(K)$ and meets precisely one wall of the first kind, and does not meet any walls of the second kind.

Observe that this definition depends on the chosen set $K$, and that if $L_{0}$ and $L_{1}$ are separated by a single wall of the first kind then they are certainly general (as in Definition 4.1). Thus, as long as there exist walls of the first kind, there will be plenty of $L_{0}$ and $L_{1}$ that satisfy this condition.

Remark 6.5. The condition imposed in Theorem 6.1 that the two lines bundles be separated by a single wall of the first kind is used only in the final step of the argument, cf. Lemma 6.20.

6.1.3. Multi-Gieseker Walls. We now describe a wall structure associated to a stability segment $\sigma(\cdot)$. This is essentially a repeat of our multi-Gieseker chamber structure from [GRT14, Section 4], but we will need something slightly more technical, so we give a self-contained account here.

Suppose that $\sigma(t)=\left(\underline{L} ; B_{1}(t), \ldots, B_{j_{0}}(t)\right)$ for $t \in[0,1]$ is a segment of stability parameters. For each $t \in[0,1]$ set

$$
\gamma_{t}:=\sum_{j} \operatorname{rank}\left(B_{j}(t)\right) c_{1}\left(L_{j}\right)^{d-1} \in N_{1}(X)_{\mathbb{R}} .
$$

So by Riemann-Roch, if $E$ is semistable with respect to $\sigma(t)$, then it is slope semistable with respect to $\gamma_{t}$.

Definition 6.6. We say that a bounded set $\mathcal{S}$ of sheaves detects the change of multi-Giesekerstability for $\sigma(\cdot)$ if the following holds: if $E$ of topological type $\tau$ and semistable with respect to $\sigma(t)$ for some $t \in[0,1]$, and $F \subset E$ is saturated with $\mu_{\gamma_{t^{\prime}}}(F) \geq \mu_{\gamma_{t^{\prime}}}(E)$ for some $t^{\prime} \in[0,1]$, then $F \in \mathcal{S}$.

Below we will apply this with $\mathcal{S}$ being a set of type $\mathcal{S}_{K}$ as in (6.3), but that is not important yet. The terminology is justified by the following:

Lemma 6.7. Let $\mathcal{S}$ be a bounded set of sheaves that detects the change of multi-Giesekerstability for a stability segment $\sigma(\cdot)$ and let $t, t^{\prime} \in[0,1]$. Suppose that for all torsion-free sheaves $E$ of topological type $\tau$ and all saturated $F \subset E$ with $F \in \mathcal{S}$ we have

$$
p_{F \subset E}^{\sigma(t)}(\leq) 0 \text { if and only if } p_{F \subset E}^{\sigma\left(t^{\prime}\right)}(\leq) 0 .
$$

Then $\sigma(t) \equiv \sigma\left(t^{\prime}\right)$.

Proof. We have seen essentially this statement before (see Lemma 4.2). Suppose that $E$ is of topological type $\tau$ and semistable with respect to $\sigma(t)$, and we show it is also (semi)stable with respect to $\sigma\left(t^{\prime}\right)$. Let $F \subset E$ be saturated. If $\mu_{\gamma_{t^{\prime}}}(F)<\mu_{\gamma_{t^{\prime}}}(E)$ then $F$ clearly does not destabilise $E$ with respect to $\sigma\left(t^{\prime}\right)$. Otherwise by definition $F \in \mathcal{S}$, and so the hypothesis that $E$ is semistable with respect to $\sigma(t)$ gives $p_{F \subset E}^{\sigma(t)}(\leq) 0$ and hence $p_{F \subset E}^{\sigma\left(t^{\prime}\right)}(\leq) 0$. 
Suppose now that $\mathcal{S}$ detects the change of multi-Gieseker-stability for $\sigma(\cdot)$. For each $F \in \mathcal{S}$ write the difference of reduced multi-Hilbert polynomials as

$$
p_{F}^{\sigma(t)}-p_{E}^{\sigma(t)}=\sum_{i=1}^{d} h_{F, i}(t) \frac{k^{d-i}}{(d-i) !},
$$

where $E$ is any sheaf of topological type $\tau$. Then, $h_{F, i}:[0,1] \rightarrow \mathbb{R}$ is linear, and thus is either identically zero or has at most 1 root in $[0,1]$.

Definition 6.8. Let $\mathcal{S}$ be a bounded set of sheaves that detects the change of multi-Giesekerstability for $\sigma(\cdot)$. We define the set of multi-Gieseker walls associated to $\mathcal{S}$ to be the set of all roots of $h_{F, i}$ for $i=1, \ldots, d$ and $F \in \mathcal{S}$ among all those $h_{F, i}$ that are not identically zero.

As each $h_{F, i}$ depends only on the topological type of $F$, the set of multi-Gieseker walls associated to $\mathcal{S}$ consists of a finite number of rational points in $[0,1]$, which we denote by $\bar{t}_{1}<$ $\ldots \bar{t}_{N}$. This divides $[0,1]$ into "chambers" within which multi-Gieseker-stability is unchanged.

Corollary 6.9. Let $\sigma(\cdot)$ be a stability segment and let $\bar{t}_{1}<\ldots<\bar{t}_{N}$ be the multi-Gieseker walls associated to a bounded set of sheaves that detects the change in multi-Gieseker-stability for $\sigma(\cdot)$. Then,

$$
\mathcal{M}_{\sigma(t)}=\mathcal{M}_{\sigma\left(t^{\prime}\right)} \text { for all } t, t^{\prime} \in\left(\bar{t}_{i}, \bar{t}_{i+1}\right) .
$$

Proof. This is immediate from Lemma 6.7, since for all saturated $F \subset E$ with $F \in \mathcal{S}$ we have $p_{F \subset E}^{\sigma(t)}(\leq) 0$ if and only if $p_{F \subset E}^{\sigma\left(t^{\prime}\right)}(\leq) 0$ by the definition of ordering of polynomials and the definition of the points $\bar{t}_{i}$.

\subsubsection{Open and (almost) perfect stability parameters.}

Definition 6.10. We say the stability segment $\sigma(\cdot)$ is open if for all $t>0$ sufficiently small we have $\sigma(0) \equiv \sigma(t)$ and $\sigma(1) \equiv \sigma(1-t)$.

The purpose of this definition is to avoid the possibility of non-trivial change of moduli space occurring at the endpoints of $[0,1]$.

Definition 6.11. Let $\mathcal{S}$ be a bounded set of sheaves. We say a stability segment $\sigma(\cdot)$ is almost perfect with respect to $\mathcal{S}$ if the following all hold:

(1) The set $\mathcal{S}$ detects the change in multi-Gieseker-stability for $\sigma(\cdot)$.

(2) $\sigma(\cdot)$ is open.

(3) We have $\mathcal{M}_{\sigma\left(t_{0}\right)} \leftrightarrow \rightarrow \mathcal{M}_{\sigma\left(t_{1}\right)}$ for all $t_{0}, t_{1} \in(0,1)$ that are not among the multi-Gieseker walls associated to $\mathcal{S}$ (cf. Definition 6.2).

\section{Remark 6.12.}

i) We did not make any requirement that we make a "minimal" choice of multi-Gieseker walls (so certainly some of the walls could be superfluous). Thus the condition (3) could, in principle, depend on the choice of set $\mathcal{S}$. We observe also that even if $\sigma(\cdot)$ is open, some of these walls could be at the endpoints of $[0,1]$; however we only demand that condition (3) holds for points in the interior of $[0,1]$.

ii) By Corollary 6.9, and since there are only a finite number of multi-Gieseker walls, we can replace condition (3) by requiring instead that $\mathcal{M}_{\sigma\left(t_{0}\right)} \leftrightarrow \rightarrow \mathcal{M}_{\sigma\left(t_{1}\right)}$ for general points $t_{0}, t_{1}$ in the two open chambers adjacent to a single wall in $(0,1)$.

iii) Furthermore, by the work done in Section 2, see especially Theorem 2.6, if $\sigma(\cdot)$ is bounded and uniform, then condition (3) certainly holds. 
iv) We reserve the terminology perfect stability segment to mean that condition (3) holds for all $t_{0}, t_{1} \in(0,1)$ including the walls, but this notion will hardly be used.

The purpose of this definition is the following obvious conclusion.

Lemma 6.13. Suppose that $\sigma(\cdot)$ is almost perfect with respect to some bounded set $\mathcal{S}$ of sheaves. Then, $\mathcal{M}_{\sigma(0)} \leftrightarrow-\rightarrow \mathcal{M}_{\sigma(1)}$.

Proof. As $\sigma(\cdot)$ is open, we have for $t>0$ sufficiently small that $\mathcal{M}_{\sigma(0)}=\mathcal{M}_{\sigma(t)}$ and $\mathcal{M}_{\sigma(1)}=$ $\mathcal{M}_{\sigma(1-t)}$. Thus, we can apply condition (3) to deduce that $\mathcal{M}_{\sigma(t)} \leftrightarrow \rightarrow \mathcal{M}_{\sigma(1-t)}$.

6.2. Strategy of the proof. The idea for the proof of Theorem 6.1 is rather natural: We first consider a stability segment $\sigma(\cdot)$ that joins two stability parameters we are interested in. In general, it will not be uniform, but it will be cut up by a finite number of rational walls. We then focus our attention on a specific wall, and find a new stability segment $\eta(\cdot)$ that joins stability parameters immediately to the left and to the right of this wall. Moreover, we arrange that this new stability segment is closer to being uniform than $\sigma(\cdot)$, in that more terms of the corresponding multi-Hilbert polynomial are independent of $t$. We then apply the same argument to $\eta(\cdot)$, and continuing in this way, we arrive at a uniform stability segment, to get the desired conclusion. Thus, since we are on a threefold, the proof divides into three steps, whose main arguments we will summarise now.

6.2.1. Step 1. Fix an open, relatively compact, connected $K \subset \operatorname{Amp}(X)_{\mathbb{R}}$, and assume $L_{0}, L_{1} \in$ Cone $^{*}(K)$ are separated by a single wall of the first kind. Without loss of generality we may assume that $L_{0}$ and $L_{1}$ are arbitrarily close to each other, and so we may assume that the line segment between $p\left(L_{0}\right)$ and $p\left(L_{1}\right)$ is completely contained in $p\left(\operatorname{Cone}^{*}(K)\right)$. Moreover, by perturbing $L_{0}$ and $L_{1}$ slightly we may, without loss of generality, assume that

$$
\begin{aligned}
\int_{X} c_{1}\left(L_{0}\right)^{3} & \neq \int_{X} c_{1}\left(L_{1}\right) c_{1}\left(L_{0}\right)^{2} \quad \text { and } \\
\int_{X} c_{1}\left(L_{1}\right)^{3} & \neq \int_{X} c_{1}\left(L_{0}\right) c_{1}\left(L_{1}\right)^{2} .
\end{aligned}
$$

Finally, by rescaling both $L_{0}$ and $L_{1}$ simultaneously we may assume they are both integral. As we have done in Section 6.1.2 above, set

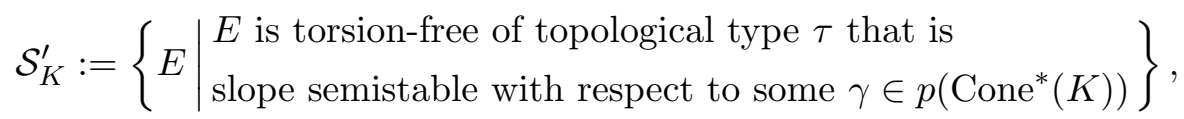

and

Now set

$$
\mathcal{S}_{K}:=\left\{\begin{array}{l|l}
F & \begin{array}{l}
F \text { is a saturated subsheaf of some } E \in \mathcal{S}_{K}^{\prime} \text { and } \\
\mu^{L}(F) \geq \mu^{L}(E) \text { for some } L \in \operatorname{Cone}^{*}(K)
\end{array}
\end{array}\right\}
$$

and consider the stability segment

$$
\sigma_{0}(t):=\frac{1-t}{\operatorname{vol}\left(L_{0}\right)}, \quad \sigma_{1}(t)=\frac{t}{\operatorname{vol}\left(L_{1}\right)}
$$

$$
\sigma(t):=\left(L_{0}, L_{1}, \sigma_{0}(t), \sigma_{1}(t)\right)
$$

Clearly, a sheaf is (semi)stable with respect to $\sigma(0)$ (resp. $\sigma(1)$ ) if and only if it is (semi)stable with respect to $L_{0}$ (resp. $L_{1}$ ). Thus, to prove Theorem 6.1 it is sufficient to prove that $\sigma(\cdot)$ is almost perfect with respect to $\mathcal{S}_{K}$, for then $\mathcal{M}_{L_{0}}=\mathcal{M}_{\sigma(0)} \leftrightarrow \rightarrow \mathcal{M}_{\sigma(1)}=\mathcal{M}_{L_{1}}$. To this end, we start with: 
Lemma 6.14. $\mathcal{S}_{K}$ detects the change of multi-Gieseker-stability for $\sigma(\cdot)$.

This, and all subsequent lemmata will be proved Section 6.4 below. The assumption that $L_{0}$ and $L_{1}$ are separated by a single wall of the first kind in particular implies they are general. Thus, by Proposition 4.3 we conclude that $\sigma(\cdot)$ is an open segment of stability parameters.

Now, since there is no reason to expect $\sigma(\cdot)$ is uniform, we have to look more closely at what happens when a wall is crossed. So, fix a wall $\bar{t} \in(0,1)$ associated to $\mathcal{S}_{K}$ and let $t_{0}$ and $t_{1}$ be general rational points in $(0,1)$ in the chamber immediately to the left and to the right of $\bar{t}$, respectively (the precise requirement for what it means for $t_{0}$ and $t_{1}$ to be general can be seen from the proof of Lemma 6.17 below). So, to show that $\sigma(\cdot)$ is almost perfect with respect to $\mathcal{S}_{K}$ it remains to show that

$$
\mathcal{M}_{\sigma\left(t_{0}\right)} \leftrightarrow \mathcal{M}_{\sigma\left(t_{1}\right)} .
$$

6.2.2. Step 2. Fix $\bar{t}, t_{0}, t_{1}$ as above, and also fix a large positive integer $a$ (to be determined below). For $s \in[0,1]$ consider the formal sum of line bundles

$$
B_{j}(s):=\sigma_{j}(\bar{t})\left(s L_{j}^{\frac{a \sigma_{j}\left(t_{1}\right)}{\sigma_{j}(\bar{t})}}+(1-s) L_{j}^{\frac{a \sigma_{j}\left(t_{0}\right)}{\sigma_{j}(\bar{t})}}\right) \quad j=1,2 .
$$

Observe that as $\bar{t} \in(0,1)$ we have $\sigma_{j}(\bar{t}) \neq 0$; moreover, for (6.9) to make sense we require that $a \sigma_{j}\left(t_{i}\right) / \sigma_{j}(\bar{t}) \in \mathbb{N}$ for $i, j=0,1$, which certainly holds for arbitrarily large $a$. Now define

$$
\eta(s):=\left(L_{0}, L_{1} ; B_{0}(s), B_{1}(s)\right) \text { for } s \in[0,1],
$$

which we observe is a stability segment, as for all $s \in[0,1]$ we have

$$
\operatorname{rank}\left(B_{0}(s)\right) \operatorname{vol}\left(L_{0}\right)+\operatorname{rank}\left(B_{1}(s)\right) \operatorname{vol}\left(L_{1}\right)=\sigma_{0}(\bar{t}) \operatorname{vol}\left(L_{0}\right)+\sigma_{1}(\bar{t}) \operatorname{vol}\left(L_{1}\right)=1 .
$$

Remark 6.15. It may help the reader to observe, more explicitly, that the multi-Hilbert polynomial of $\eta(s)$ for a sheaf $E$ is given by

$$
\begin{aligned}
P_{E}^{\eta(s)}(k)= & \frac{1-\bar{t}}{\operatorname{vol}\left(L_{0}\right)} s \chi\left(E \otimes L_{0}^{k} \otimes L_{0}^{\frac{a\left(1-t_{1}\right)}{1-\bar{t}}}\right)+\frac{1-\bar{t}}{\operatorname{vol}\left(L_{0}\right)}(1-s) \chi\left(E \otimes L_{0}^{k} \otimes L_{0}^{\frac{a\left(1-t_{0}\right)}{1-\bar{t}}}\right) \\
& +\frac{\bar{t}}{\operatorname{vol}\left(L_{1}\right)} s \chi\left(E \otimes L_{1}^{k} \otimes L_{1}^{\frac{a t_{1}}{\bar{t}}}\right)+\frac{\bar{t}}{\operatorname{vol}\left(L_{1}\right)}(1-s) \chi\left(E \otimes L_{1}^{k} \otimes L_{1}^{\frac{a t_{0}}{\bar{t}}}\right) .
\end{aligned}
$$

There are two reasons for this particular choice of twisting. First, from Riemann-Roch one can verify that the part of the $k^{2}$ coefficient of $P_{E}^{\eta(s)}$ that depends on $E$ is independent of $s$ (this essentially follows as $\operatorname{rank}\left(B_{j}(s)\right)$ is independent of $s$ ). So, even though $\eta(\cdot)$ may not be a uniform segment of stability parameters, it is closer to being uniform than $\sigma(\cdot)$ was. Second, at the endpoints $s=0$ and $s=1$ we get back the stability with respect to $\sigma\left(t_{0}\right)$ and $\sigma\left(t_{1}\right)$, as made precise in the following lemma which is adapted from ideas of MatsukiWentworth [MW97, Thm 4.1].

Lemma 6.16. For $a \in \mathbb{N}$ sufficiently large, $\eta(0) \equiv \sigma\left(t_{0}\right)$ and $\eta(1) \equiv \sigma\left(t_{1}\right)$.

As a consequence, our goal becomes to show that $\eta(\cdot)$ is almost perfect with respect to $\mathcal{S}_{K}$, for then $\mathcal{M}_{\sigma\left(t_{0}\right)}=\mathcal{M}_{\eta(0)} \leftrightarrow \rightarrow \mathcal{M}_{\eta(1)}=\mathcal{M}_{\sigma\left(t_{1}\right)}$. Towards this goal we start with:

\section{Lemma 6.17.}

(1) $\mathcal{S}_{K}$ detects the change of multi-Gieseker-stability for $\eta(\cdot)$.

(2) For $a \in \mathbb{N}$ sufficiently large, $\eta(\cdot)$ is an open segment of stability parameters. 
Now, fix a multi-Gieseker wall $\bar{s} \in(0,1)$ for $\eta(\cdot)$ associated to $\mathcal{S}_{K}$ and let $s_{0}$ and $s_{1}$ be points in $(0,1)$ contained in adjacent chambers located immediately either side of this wall. So, to prove $\eta(\cdot)$ is almost perfect with respect to $\mathcal{S}_{K}$, it remains to prove

$$
\mathcal{M}_{\eta\left(s_{0}\right)} \leftrightarrow \rightarrow \mathcal{M}_{\eta\left(s_{1}\right)} \text {. }
$$

6.2.3. Step 3. Consider a fixed $\bar{s}, s_{0}$ and $s_{1}$ as above. The third, and final, stability segment that we need is built from the following elementary, but tedious, construction:

Lemma 6.18. For sufficiently large $\lambda \in \mathbb{R}$ and all $b \in \mathbb{R}_{>0}$, for $r \in[0,1]$ there exist formal sums of line bundles $D_{1}(r)$ and $D_{2}(r)$ whose coefficients are linear in $r$ and strictly positive for $r \in(0,1)$ such that the following hold:

(1) $\operatorname{rank}\left(D_{j}(r)\right)=\operatorname{rank}\left(B_{j}(\bar{s})\right)$ and $c_{1}\left(D_{j}(r)\right)=c_{1}\left(B_{j}(\bar{s})\right)$ (and so in particular both are independent of $r)$.

(2) For $i=0,1$ we have

$$
\sum_{j=0}^{1} \operatorname{ch}_{2}\left(D_{j}(i)\right)=b \sum_{j=0}^{1} \operatorname{rank}\left(B_{j}(\bar{s})\right)\left(\lambda+\frac{a\left(s_{i}-\bar{s}\right)\left(\sigma_{j}\left(t_{1}\right)-\sigma_{j}\left(t_{0}\right)\right)}{\sigma_{j}(\bar{t})}\right) c_{1}\left(L_{j}\right)^{2}+\sum_{j=0}^{1} \operatorname{ch}_{2}\left(B_{j}(\bar{s})\right)
$$

(3) The quantity $\sum_{j=0}^{1} c_{1}\left(L_{j}\right) \operatorname{ch}_{2}\left(D_{j}(r)\right)$ is independent of $r$.

Here, we have extended the definition of Chern character linearly to formal sum of line bundles; so, if $A_{j i}$ are line bundles, $\operatorname{ch}\left(\sum_{i} a_{j i} A_{j i}\right):=\sum_{i} a_{j i} \operatorname{ch}\left(A_{j i}\right) \in H^{*}(X, \mathbb{R})$. In particular, $\operatorname{rank}\left(\sum_{i} a_{j i} A_{j i}\right)=\sum_{i} a_{j i}$ as defined before.

Granted this, consider

$$
\zeta(r):=\left(L_{0}, L_{1} ; D_{0}(r), D_{1}(r)\right) \text { for } r \in[0,1] .
$$

\section{Lemma 6.19.}

(1) $\mathcal{S}_{K}$ detects the change of multi-Gieseker-stability for $\zeta(\cdot)$,

(2) $\zeta(\cdot)$ is a bounded and uniform segment of stability parameters, and

(3) for $b \in \mathbb{R}_{>0}$ sufficiently large, $\zeta(0) \equiv \eta\left(s_{0}\right)$ and $\zeta(1) \equiv \eta\left(s_{1}\right)$.

Lemma 6.20. For $b \in \mathbb{R}_{>0}$ sufficiently large, $\zeta(\cdot)$ is open, and thus perfect.

Thus, by Theorem 2.6 the moduli spaces defined by the endpoints of $\zeta$ are related by a finite number of Thaddeus-flips through moduli spaces of sheaves. That is,

$$
\mathcal{M}_{\eta\left(s_{0}\right)}=\mathcal{M}_{\zeta(0)} \leftrightarrow \rightarrow \mathcal{M}_{\zeta(1)}=\mathcal{M}_{\eta\left(s_{1}\right)} .
$$

This proves (6.10), which in turn proves (6.8), which finally proves Theorem 6.1.

6.3. Surfaces. If $X$ is a surface the ample cone $\operatorname{Amp}(X)$ is divided into chambers by a locally finite collection of rational linear walls that witness the change in slope stability. Suppose that $L_{0}, L_{1} \in \operatorname{Amp}_{\mathbb{Z}}(X)$ lie in the interior of adjacent chambers separated only by a single wall, so for each wall $W$ the straight line through $L_{0}$ and $L_{1}$ is either contained in $W$ or meets it at a single point (necessarily rational) which we denote by $\bar{L}$. We may assume that $\bar{L}$ is the midpoint of this segment, and by rescaling we may as well take it to be integral. For a large positive integer $a$, consider the stability segment given by

$$
\sigma(t)=\left(\bar{L}, \bar{L} ; \frac{t}{\operatorname{vol}(\bar{L})} L_{1}^{a}, \frac{1-t}{\operatorname{vol}(\bar{L})} L_{0}^{a}\right), \quad t \in[0,1]
$$

Using the same techniques as described above, one checks this is a uniform segment of stability parameters, and moreover if $a$ is sufficiently large any sheaf $E$ of given topological type is 
(semi)stable with respect to $\sigma(0)$ (resp. $\sigma(1), \sigma(1 / 2)$ ) if and only if it is Gieseker-(semi)stable with respect to $L_{0}\left(\right.$ resp. $\left.L_{1}, \bar{L}\right)$ (compare [MW97, Sect. 3]). Hence, we may conclude from Theorem 2.6 that $\mathcal{M}_{L_{0}}, \mathcal{M}_{L_{1}}$, and $\mathcal{M}_{\bar{L}}$ are related to each other through a finite number of Thaddeus-flips through moduli spaces of sheaves. Since this holds for any two such $L_{0}$ and $L_{1}$, one may apply this statement repeatedly to deduce the same holds for any two moduli spaces of Gieseker-semistable torsion-free sheaves on a smooth surface.

6.4. Proofs of the lemmata. In this section, we prove Theorem 6.1 by establishing the lemmata stated in the previous section. The subsequent proofs involve Riemann-Roch computations that for convenience we state here.

Suppose that

$$
\sigma=\left(L_{0}, L_{1} ; B_{0}, B_{1}\right)
$$

where $B_{i}$ is a formal sum of line bundles as in (1.2) (whose coefficients could depend on a parameter) and assume

$$
\operatorname{rank}\left(B_{0}\right) \operatorname{vol}\left(L_{0}\right)+\operatorname{rank}\left(B_{1}\right) \operatorname{vol}\left(L_{1}\right)=1 .
$$

Then, by Riemann-Roch, if $E$ is a non-trivial torsion-free sheaf on our threefold $X$, the multiplicity of $E$ is $r_{E}^{\sigma}=\operatorname{rank}(E)$, and if $F \subset E$ is a non-trivial subsheaf, then

$$
p_{F \subset E}^{\sigma}=\left\langle\left\langle h_{1}\left\|h_{2}\right\| h_{3}\right\rangle\right\rangle,
$$

where

$$
\begin{aligned}
h_{1} & =\operatorname{hilb}_{1}(F, E) \cdot \sum \operatorname{rank}\left(B_{j}\right) c_{1}\left(L_{j}\right)^{2}, \\
h_{2} & =\operatorname{hilb}_{2}(F, E) \cdot \sum \operatorname{rank}\left(B_{j}\right) c_{1}\left(L_{j}\right)+\operatorname{hilb}_{1}(F, E) \cdot \sum c_{1}\left(L_{j}\right) c_{1}\left(B_{j}\right), \\
h_{3} & =\operatorname{hilb}_{3}(F, E) \cdot \sum \operatorname{rank}\left(B_{j}\right)+\operatorname{hilb}_{2}(F, E) . \sum c_{1}\left(B_{j}\right)+\operatorname{hilb}_{1}(F, E) . \sum \operatorname{ch}_{2}\left(B_{j}\right),
\end{aligned}
$$

the sums being over $j=0,1$.

Proof of Lemma 6.14. We have to show $\mathcal{S}_{K}$ detects the change of multi-Gieseker-stability for $\sigma(\cdot)$. By definition $\sigma(t)=\left(L_{0}, L_{1} ; \sigma_{0}(t), \sigma_{1}(t)\right)=\left(L_{0}, L_{1} ; \sigma_{0}(t) \mathcal{O}_{X}, \sigma_{1}(t) \mathcal{O}_{X}\right)$. Thus, as in (6.4), we must consider the curve class

$$
\gamma_{t}=\sigma_{0}(t) c_{1}\left(L_{0}\right)^{2}+\sigma_{1}(t) c_{1}\left(L_{1}\right)^{2} \text { for } t \in[0,1] .
$$

Observe that since we assumed the line segment between $p\left(L_{0}\right)$ and $p\left(L_{1}\right)$ lies completely within $p\left(\operatorname{Cone}^{*}(K)\right)$, we have that $\gamma_{t} \in p\left(\operatorname{Cone}^{*}(K)\right)$ for all $t \in[0,1]$.

Suppose that $E$ is of topological type $\tau$ and semistable with respect to $\sigma(t)$ for some $t \in[0,1]$. Then, it is slope semistable with respect to $\gamma_{t}$, so $E \in \mathcal{S}_{K}^{\prime}$ (as defined in (6.6)). Suppose $F \subset E$ is saturated and $\mu_{\gamma_{t^{\prime}}}(F) \geq \mu_{\gamma_{t^{\prime}}}(E)$ for some $t^{\prime} \in[0,1]$. Then, letting $L^{\prime} \in \operatorname{Cone}^{*}(K)$ be such that $p\left(L^{\prime}\right)=\gamma_{t^{\prime}}$ we have $\mu^{L^{\prime}}(F) \geq \mu^{L^{\prime}}(E)$. Thus, $F \in \mathcal{S}_{K}$ (as defined in (6.7)). Therefore, $\mathcal{S}_{K}$ detects the change in change of multi-Gieseker-stability for $\sigma(\cdot)$ as claimed.

For later use, we record that if $F \subset E$, then by Riemann-Roch (6.11)

$$
p_{F \subset E}^{\sigma(t)}=\left\langle\left\langle h_{1}(t)|| h_{2}(t)|| h_{3}(t)\right\rangle\right\rangle,
$$

where

$$
h_{i}(t)=\operatorname{hilb}_{i}(F, E) . \sum_{j} \sigma_{j}(t) c_{1}\left(L_{j}\right)^{3-i}
$$


Proof of Lemma 6.17. We have to show (1) $\mathcal{S}_{K}$ detects the change of multi-Gieseker-stability for $\eta(\cdot)$ and (2) For $a \in \mathbb{N}$ sufficiently large, $\eta(\cdot)$ is an open segment of stability parameters.

The proof of (1) is easy. We recall by definition, see (6.9), $\eta(s)=\left(L_{0}, L_{1} ; B_{0}(s), B_{1}(s)\right)$, where

$$
B_{j}(s)=\sigma_{j}(\bar{t})\left(s L_{j}^{\frac{a \sigma_{j}\left(t_{1}\right)}{\sigma_{j}(\bar{t})}}+(1-s) L_{j}^{\frac{a \sigma_{j}\left(t_{0}\right)}{\sigma_{j}(\bar{t})}}\right) .
$$

Hence,

$$
\begin{aligned}
\operatorname{rank}\left(B_{j}(s)\right) & =\sigma_{j}(\bar{t}), \\
c_{1}\left(B_{j}(s)\right) & =a\left(s \sigma_{j}\left(t_{1}\right)+(1-s) \sigma_{j}\left(t_{0}\right)\right) c_{1}\left(L_{j}\right) \\
& =a \sigma_{j}\left(s t_{1}+(1-s) t_{0}\right) c_{1}\left(L_{j}\right), \\
\operatorname{ch}_{2}\left(B_{j}(s)\right) & =\frac{a^{2}}{2 \sigma_{j}(\bar{t})}\left(s \sigma_{j}\left(t_{1}\right)^{2}+(1-s) \sigma_{j}\left(t_{0}\right)^{2}\right) c_{1}\left(L_{j}\right)^{2} .
\end{aligned}
$$

Hence, to show $\mathcal{S}_{K}$ witnesses the change in multi-Gieseker-stability for $\eta(\cdot)$ we are required to look at the curve class

$$
\gamma_{\bar{t}}:=\sigma_{0}(\bar{t}) c_{1}\left(L_{0}\right)^{2}+\sigma_{1}(\bar{t}) c_{1}\left(L_{1}\right)^{2}
$$

(which actually is independent of $s$ ). Now, just as in the proof of Lemma 6.14, in which we showed that $\mathcal{S}_{K}$ witnesses the change in multi-Gieseker-stability for $\sigma(\cdot)$, we have $\gamma_{\bar{t}} \in$ $p\left(\right.$ Cone* $\left.^{*}(K)\right)$. Thus, exactly the same proof shows that $\mathcal{S}_{K}$ detects the change in multiGieseker-stability for $\eta(\cdot)$.

We now turn to proving that for $a \in \mathbb{N}$ sufficiently large, $\eta(\cdot)$ is an open segment of stability parameters. By Lemma 6.7 it is sufficient to show that for $s>0$ sufficiently small, if $E$ is torsion-free of topological type $\tau$ and $F \subset E$ is saturated with $F \in \mathcal{S}_{K}$, we have

$$
p_{F \subset E}^{\eta(0)}(\leq) 0 \text { if and only if } p_{F \subset E}^{\eta(s)}(\leq) 0
$$

and

$$
p_{F \subset E}^{\eta(1)}(\leq) 0 \text { if and only if } p_{F \subset E}^{\eta(1-s)}(\leq) 0 .
$$

We will prove the first of these, as the proof of the second is identical. Let $F \subset E$ be saturated with $F \in \mathcal{S}_{K}$ and $E$ torsion-free of topological type $\tau$. Then, using Riemann-Roch (6.11) we have

where

$$
p_{F \subset E}^{\eta(s)}=\left\langle\left\langle u_{1}\left\|u_{2}(s)\right\| u_{3}(s)\right\rangle\right\rangle
$$

$$
\begin{aligned}
u_{1} & =h_{1}(\bar{t}), \\
u_{2}(s) & =h_{2}(\bar{t})+a h_{1}\left(s t_{1}+(1-s) t_{0}\right), \\
u_{3}(s) & =h_{3}(\bar{t})+a h_{2}\left(s t_{1}+(1-s) t_{0}\right)+a^{2} \epsilon(s),
\end{aligned}
$$

with $h_{i}(\cdot)$ defined as in (6.14) and

$$
\epsilon(s):=\frac{1}{2} \operatorname{hilb}_{1}(F, E) \cdot \sum_{j} \frac{s \sigma_{j}\left(t_{1}\right)^{2}+(1-s) \sigma_{j}\left(t_{0}\right)^{2}}{\sigma_{j}(\bar{t})} c_{1}\left(L_{j}\right)^{2} .
$$

We claim that for $i=1,2,3$ we either have $u_{i}(s) \equiv 0$ for all $s \in[0,1]$ or $u_{i}(0) \neq 0$. Observe that this claim proves (6.17) (and thus completes the proof of the lemma), since we can take $s>0$ sufficiently small so there are no roots of $u_{i}$ in $[0, s]$ for all (non-trivial) such $u_{i}$, and then (6.17) follows from the definition of ordering of polynomials in terms of the lexicographic order on the coefficients.

Now the claim is clear for $u_{1}$ as it is independent of $s$. For $u_{2}$, notice that if $u_{2}(0)=0$, then $h_{2}(\bar{t})+a h_{1}\left(t_{0}\right)=0$. So, as $a$ can be chosen arbitrarily large, this implies $h_{1}\left(t_{0}\right)=0$ and $h_{2}(\bar{t})=0$. However, $t_{0}$ was chosen to be a point in an open chamber for $\sigma(\cdot)$, so $h_{1}\left(t_{0}\right)=0$ 
implies $h_{1} \equiv 0$. Looking back at the expression (6.19) for $u_{2}$, we then conclude that $u_{2}$ is actually independent of $s$, which proves the claim for $u_{2}$.

A similar argument works for $u_{3}$. Suppose that

$$
0=u_{3}(0)=h_{3}(\bar{t})+a h_{2}\left(t_{0}\right)+a^{2} \epsilon(0) .
$$

Then, as $a$ can be chosen arbitrarily large, we must have $\epsilon(0)=0$, which means

$$
0=\operatorname{hilb}_{1}(F, E)\left(\frac{\left(1-t_{0}\right)^{2}}{\operatorname{vol}\left(L_{0}\right)(1-\bar{t})} c_{1}\left(L_{0}\right)^{2}+\frac{t_{0}^{2}}{\operatorname{vol}\left(L_{1}\right) \bar{t}} c_{1}\left(L_{1}\right)^{2}\right) .
$$

Think of this as a quadratic polynomial in $t_{0}$, whose linear term is

$$
-\frac{2}{\operatorname{vol}\left(L_{0}\right)(1-\bar{t})} \operatorname{hilb}_{1}(F, E) c_{1}\left(L_{0}\right)^{2} .
$$

Then, by the assumption that $L_{0}$ does not lie on any wall in $\tilde{\mathcal{W}}_{K}$, either hilb $(F, E) c_{1}(L)^{2}=0$ for all $L \in \operatorname{Amp}(X)_{\mathbb{R}}$ (in which case $\epsilon(\cdot) \equiv 0$ ), or this linear term is non-trivial, and so $\epsilon(0)$ is non-constant in $t_{0}$. Thus, as $t_{0}$ was assumed to be a general point in the open chamber adjacent to $\bar{t}$, we may as well assume it was chosen so $\epsilon(0) \neq 0$, which is absurd. Moreover, as there are only a finite number of topological types among all the sheaves $F \subset E$ under consideration, due to boundedness of $\mathcal{S}_{K}$, we can make such a choice uniformly over all such pairs.

Thus, we must in fact have $\epsilon \equiv 0$. So, looking back at the expression for $u_{3}$, the assumption $u_{3}(0)=0$ is $0=h_{3}(\bar{t})+a h_{2}\left(t_{0}\right)$ and hence $h_{2}\left(t_{0}\right)=0$ as $a$ is assumed to be arbitrarily large. As $t_{0}$ is general in the open chamber, this implies $h_{2}(t)=0$ for all $t \in[0,1]$, at which point one observes $u_{3}$ is also independent of $s$, which proves the claim for $u_{3}$.

Proof of Lemma 6.16. We shall show that $\eta(0) \equiv \sigma\left(t_{0}\right)$ (the other statement being proved similarly). We first establish the following

Claim: It is sufficient to prove that if $E$ is of topological type $\tau$ and $F \subset E$ is saturated with $F \in \mathcal{S}_{K}$, then

$$
p_{F \subset E}^{\sigma\left(t_{0}\right)}(\leq) 0 \text { if and only if } p_{F \subset E}^{\eta(0)}(\leq) 0
$$

We have seen essentially this argument before: Assume (6.20) holds and suppose that $E$ is (semi)stable with respect to $\sigma\left(t_{0}\right)$. Then, it is slope semistable with respect to $\gamma_{t_{0}}(6.12)$, and so $E \in \mathcal{S}_{K}^{\prime}$. We wish to show that $E$ is (semi)stable with respect to $\eta(0)$. So, suppose there exists a saturated subsheaf $F \subset E$ such that $p_{F \subset E}^{\eta(0)}>0$. Then, $F \in \mathcal{S}_{K}$ since $\mathcal{S}_{K}$ detects the change in multi-Gieseker-stability for $\eta(\cdot)$. Hence, by condition (6.20) we have $p_{F \subset E}^{\sigma\left(t_{0}\right)}>0$, which is a contradiction. The converse is proved similarly.

So, we proceed to prove (6.20) for any such sheaves $F$ and E. From (6.13), (6.18), (6.19) we have

and

$$
p_{F \subset E}^{\sigma\left(t_{0}\right)}=\left\langle\left\langle h_{1}\left(t_{0}\right)\left\|h_{2}\left(t_{0}\right)\right\| h_{3}\left(t_{0}\right)\right\rangle\right\rangle,
$$

$$
\begin{aligned}
p_{F \subset E}^{\eta(0)} & =\left\langle\left\langle u_{1}\left\|u_{2}(0)\right\| u_{3}(0)\right\rangle\right\rangle \\
& =\left\langle\left\langle h_{1}(\bar{t})\left\|h_{2}(\bar{t})+a h_{1}\left(t_{0}\right)\right\| h_{3}(\bar{t})+a h_{2}\left(t_{0}\right)+\operatorname{hilb}_{1}(F, E) \cdot \epsilon\right\rangle\right\rangle,
\end{aligned}
$$

where $h_{j}(\cdot)$ is as in (6.14), and $\epsilon$ is a linear combination of $c_{1}\left(L_{0}\right)^{2}$ and $c_{1}\left(L_{1}\right)^{2}$.

Assume $p_{F \subset E}^{\eta(0)}(\leq) 0$ and we shall show that $p_{F \subset E}^{\sigma\left(t_{0}\right)}(\leq) 0$. If $h_{1}(\bar{t})<0$, then $h_{1}\left(t_{0}\right)<0$, since we are assuming that $t_{0}$ lies in an open chamber immediately adjacent to $\bar{t}$. Thus, we also have 
$p_{F \subset E}^{\sigma\left(t_{0}\right)}(\leq) 0$ in this case. If $h_{1}(\bar{t})=0$ and $h_{2}(\bar{t})+a h_{1}\left(t_{0}\right)<0$, then by making $a$ sufficiently large we get either $h_{1}\left(t_{0}\right)<0$, in which case we are done, or $h_{1}\left(t_{0}\right)=0$ and $h_{2}(\bar{t})<0$, in which case $h_{1} \equiv 0$ and $h_{2}(\bar{t})<0$. But then $h_{2}\left(t_{0}\right)<0$, since $t_{0}$ lies in an open chamber immediately adjacent to $\bar{t}$, and we are done again. So, assume now that $h_{1} \equiv 0, h_{2} \equiv 0$. Then, $\operatorname{hilb}_{1}(F, E) . \epsilon=0$ as well and the implication follows from the fact that $h_{3}(t)$ has constant sign on $[0,1]$.

For the converse, if $h_{1}\left(t_{0}\right)<0$, then $h_{1}(\bar{t}) \leq 0$ since $\bar{t}$ lies in the closure of the chamber containing $t_{0}$, and $h_{2}(\bar{t})+a h_{1}\left(t_{0}\right)(\leq) 0$ for sufficiently large $a$. If $h_{1}\left(t_{0}\right)=0$. then $h_{1} \equiv 0$, as $t_{0}$ is in an open chamber, which in turn implies $\operatorname{hilb}_{1}(F, E) . \epsilon=0$. We deal now with the conditions $h_{2}\left(t_{0}\right)<0$ and $h_{2}\left(t_{0}\right)=0$ in the same way we have dealt with $h_{1}\left(t_{0}\right)<0$ and $h_{1}\left(t_{0}\right)=0$. Observe that the size of $a$ needed depends only on the topological type of $F$, and since there are only finitely many types of $F$ under consideration we can pick such an integer $a$ uniformly.

Remark 6.21. The previous two proofs can be simplified at a cost of making the stability segment more complicated. More precisely, if we arrange for the $B_{j}(s)$ to have the same rank and first Chern class as before, but with $\operatorname{ch}_{2}\left(B_{j}\right)=\lambda \sigma_{j}(\bar{t})$ for some $\lambda \in \mathbb{R}$. Then $\epsilon \equiv \lambda h_{1}(\bar{t})$ (so in particular is constant in $s$ ). We leave it as an exercise to the reader to prove that one can construct such $B_{j}(s)$ of the form $\frac{1}{i_{0}} \sigma_{j}(\bar{t}) \sum_{i=1}^{i_{0}} L_{j}^{a_{i j}}$ for suitable $a_{i j} \in \mathbb{Z}$. We have chosen to use the simpler stability segment as we consider it more natural.

Our next task is to construct the necessary twisting for the stability segment $\zeta(\cdot)$.

Lemma 6.22. Let $\lambda, \mu \in \mathbb{R}_{>0}$ and $A$ be a line bundle. Then, there exists a formal sum $B$ of line bundles with positive coefficients such that $\operatorname{rank}(B)=\lambda, c_{1}(B)=0$ and $\operatorname{ch}_{2}(B)=$ $\mu c_{1}(A)^{2}$.

Proof. Let $\alpha:=\mu n^{-2}$, where $n$ is an integer large enough so $\alpha<\lambda / 2$. Then,

$$
B:=\alpha\left(A^{n}+A^{-n}\right)+(\lambda-2 \alpha) \mathcal{O}_{X}
$$

has the desired properties.

Corollary 6.23. Let $L_{0}$ and $L_{1}$ be line bundles and $\alpha_{0}, \alpha_{1} \in \mathbb{R}_{>0}$. Then, there exists a formal sum $C$ of line bundles with positive coefficients such that $\operatorname{rank}(C)=1, c_{1}(C)=0$, and

$$
\operatorname{ch}_{2}(C)=\sum_{k=0}^{1} \alpha_{k} c_{1}\left(L_{k}\right)^{2}
$$

Proof. Apply the previous lemma twice to get $C_{k}$ with $\operatorname{rank}\left(C_{k}\right)=1 / 2, c_{1}\left(C_{k}\right)=0$, and $\operatorname{ch}_{2}\left(C_{k}\right)=\alpha_{k} c_{1}\left(L_{k}\right)^{2}$. Then, set $C:=C_{1}+C_{2}$.

Lemma 6.24. Let $q_{j i} \in \mathbb{R}$ for $j, i=0,1$ and $r_{0}, r_{1} \in \mathbb{R}_{>0}$. Suppose also $c_{j i} \in \mathbb{R}$ with $c_{0 k} \neq c_{1 k}$ for $k \in\{0,1\}$. Then, for $\lambda \gg 0$ and all $b>0$, there exist $\alpha_{j i k} \in \mathbb{R}_{>0}$ such that

$$
\begin{aligned}
\sum_{j} r_{j} \alpha_{j i k} & =r_{k} b\left(\lambda+q_{k i}\right) & & \text { for } i, k=0,1 \\
r_{0} c_{0 k}\left(\alpha_{01 k}-\alpha_{00 k}\right) & =r_{1} c_{1 k}\left(\alpha_{10 k}-\alpha_{11 k}\right) & & \text { for } k=0,1 .
\end{aligned}
$$

Proof. The equations for $k=0$ and $k=1$ separate, so it is sufficient to prove the claim for $k=0$. By linear trivialities, since $c_{00} \neq c_{10}$, there certainly exist $\beta_{j i} \in \mathbb{R}$ with

$$
\begin{aligned}
r_{0} \beta_{00}+r_{1} \beta_{10} & =r_{0} q_{00} \\
r_{0} \beta_{01}+r_{1} \beta_{11} & =r_{0} q_{01} \\
r_{0} c_{00}\left(\beta_{01}-\beta_{00}\right) & =c_{10} r_{1}\left(\beta_{10}-\beta_{11}\right) .
\end{aligned}
$$


Now, let $\alpha_{0 i 0}=b\left(\beta_{0 i}+\lambda / 2\right)$ and $\alpha_{1 i 0}=b\left(\beta_{1 i}+\lambda r_{0} / 2 r_{1}\right)$ for $i=0,1$ which are positive as long as $\lambda$ is sufficiently large.

Proof of Lemma 6.18. Set $r_{j}:=\operatorname{rank}\left(B_{j}(\bar{s})\right)=\sigma_{j}(\bar{t})$ and

$$
q_{j i}:=\frac{a\left(s_{i}-\bar{s}\right)\left(\sigma_{j}\left(t_{1}\right)-\sigma_{j}\left(t_{0}\right)\right)}{\sigma_{j}(\bar{t})} .
$$

Then, recall we are to find $D_{j}(r)$ such that

(1) $\operatorname{rank}\left(D_{j}(r)\right)=r_{j}$ and $c_{1}\left(D_{j}(r)\right)=c_{1}\left(B_{j}(\bar{s})\right)$.

(2) For $i=0,1$, we have

$$
\sum_{j} \operatorname{ch}_{2}\left(D_{j}(i)\right)=b \sum_{j} r_{j}\left(\lambda+q_{j i}\right) c_{1}\left(L_{j}\right)^{2}+\sum_{j} \operatorname{ch}_{2}\left(B_{j}(\bar{s})\right) .
$$

(3) The quantity $\sum_{j} c_{1}\left(L_{j}\right) \operatorname{ch}_{2}\left(D_{j}(r)\right)$ is independent of $r$.

To do this, let $c_{i k}=\int_{X} c_{1}\left(L_{i}\right) c_{1}\left(L_{k}\right)^{2}$. Then, our assumption (6.5) says that $c_{0 k} \neq c_{1 k}$ for $k=0,1$. So, we can apply Lemma 6.24 to get $\alpha_{j i k}>0$ for $i, j, k \in\{0,1\}$ that satisfy (6.21) and (6.22). From Corollary 6.23 there exist formal sums of line bundles $C_{j i}$ with positive coefficients, such that $\operatorname{rank}\left(C_{j i}\right)=1$ and $c_{1}\left(C_{j i}\right)=0$ and

$$
\operatorname{ch}_{2}\left(C_{j i}\right)=\alpha_{j i 0} c_{1}\left(L_{0}\right)^{2}+\alpha_{j i 1} c_{1}\left(L_{1}\right)^{2}=\sum_{k} \alpha_{j i k} c_{1}\left(L_{k}\right)^{2} .
$$

Setting

$$
D_{j}(r):=B_{j}(\bar{s}) \otimes\left((1-r) C_{j 0}+r C_{j 1}\right)
$$

one sees immediately that property (1) holds. For (2), we compute

$$
\begin{aligned}
\sum_{j} \operatorname{ch}_{2}\left(D_{j}(0)\right) & =\sum_{j} \operatorname{ch}_{2}\left(B_{j}(\bar{s}) \otimes C_{j 0}\right) \\
& =\sum_{j} r_{j} \operatorname{ch}_{2}\left(C_{j 0}\right)+\operatorname{ch}_{2}\left(B_{j}(\bar{s})\right) \\
& =\sum_{j} r_{j}\left(\alpha_{j 00} c_{1}\left(L_{0}\right)^{2}+\alpha_{j 01} c_{1}\left(L_{1}\right)^{2}\right)+\sum_{j} \operatorname{ch}_{2}\left(B_{j}(\bar{s}) .\right.
\end{aligned}
$$

With (6.21) this yields property (2) when $i=0$ (and the computation for $i=1$ is the same). Finally,

$$
\begin{aligned}
\sum_{j} c_{1}\left(L_{j}\right) \operatorname{ch}_{2}\left(D_{j}(r)\right) & =\sum_{j} c_{1}\left(L_{j}\right) \cdot\left(\operatorname{ch}_{2}\left(B_{j}(\bar{s})\right)+r_{j}\left((1-r) \operatorname{ch}_{2}\left(C_{j 0}\right)+r \operatorname{ch}_{2}\left(C_{j 1}\right)\right)\right) \\
& =r \sum_{j} r_{j} c_{1}\left(L_{j}\right) \cdot\left(\operatorname{ch}_{2}\left(C_{j 1}\right)-\operatorname{ch}_{2}\left(C_{j 0}\right)\right)+\epsilon
\end{aligned}
$$

where $\epsilon$ is independent of $r$. But using (6.23) and (6.21),

$$
\sum_{j} r_{j} c_{1}\left(L_{j}\right) \cdot\left(\operatorname{ch}_{2}\left(C_{j 1}\right)-\operatorname{ch}_{2}\left(C_{j 0}\right)\right)=\sum_{k} \sum_{j} r_{j}\left(\alpha_{j 1 k}-\alpha_{j 0 k}\right) c_{j k}=0
$$

which gives property (3).

Proof of Lemma 6.19. We have to show that (1) $\mathcal{S}_{K}$ detects the change in multi-Gieseker stability for $\zeta(\cdot),(2) \zeta(\cdot)$ is a bounded and uniform segment of stability parameters and (3) for $b \in \mathbb{R}_{>0}$ sufficiently large, $\zeta(0) \equiv \eta\left(s_{0}\right)$ and $\zeta(1) \equiv \eta\left(s_{1}\right)$.

We recall that by construction $\zeta(r)=\left(L_{0}, L_{1} ; D_{0}(r), D_{1}(r)\right)$ for $r \in[0,1]$, where

$$
\operatorname{rank}\left(D_{j}(r)\right)=\operatorname{rank}\left(B_{j}(\bar{s})\right)=r_{j} \text { and } c_{1}\left(D_{j}(r)\right)=c_{1}\left(B_{j}(\bar{s})\right)
$$

In particular,

$$
\operatorname{vol}\left(L_{0}\right) \operatorname{rank}\left(D_{0}(r)\right)+\operatorname{vol}\left(L_{1}\right) \operatorname{rank}\left(D_{1}(r)\right)=\operatorname{vol}\left(L_{0}\right) \operatorname{rank}\left(B_{0}(\bar{s})\right)+\operatorname{vol}\left(L_{1}\right) \operatorname{rank}\left(B_{1}(\bar{s})\right)=1,
$$
so $\zeta(r)$ is in fact a stability segment. 
Clearly, $\zeta(\cdot)$ is bounded as any sheaf that is semistable with respect to $\zeta(r)$ for some $r \in[0,1]$ is slope semistable with respect to $\gamma_{\bar{t}}$. Moreover, from this (1) follows in the same way as in the proof of Lemma 6.17. We next show that $\zeta(\cdot)$ is uniform. To see this, let $E$ be torsion-free, so by Riemann-Roch

$$
P_{E}^{\zeta(r)}(k)=\operatorname{rank}(E) k^{3}+a_{1} k^{2}+a_{2} k+a_{3}
$$

with

$$
\begin{gathered}
a_{1}=\sum_{j} c_{1}\left(L_{j}\right)^{2} \cdot\left(r_{j} \operatorname{Hilb}_{1}(E)+\operatorname{rank}(E) c_{1}\left(D_{j}(r)\right)\right. \\
a_{2}=\sum_{j} c_{1}\left(L_{j}\right) \cdot\left[r_{j} \operatorname{Hilb}_{2}(E)+\operatorname{rank}(E) \operatorname{ch}_{2}\left(D_{j}(r)+c_{1}(E) c_{1}\left(D_{j}(r)\right)\right.\right. \\
\left.+\operatorname{rank}(E) c_{1}\left(D_{j}(r)\right) \operatorname{Todd}_{1}(X)\right] .
\end{gathered}
$$

Now, by (6.24) we see $r_{j}=\sigma_{k}(\bar{t})$ and $c_{1}\left(D_{j}(r)\right)$ are independent of $r$, and so $a_{1}$ is independent of $r$. Moreover, by Lemma 6.18(3) the quantity $\sum_{j} c_{1}\left(L_{j}\right) \operatorname{ch}_{2}\left(D_{j}(r)\right)$ is also independent of $r$, and hence so is $a_{2}$. Thus, the only term that depends non-trivially on $r$ is $a_{3}$, and thus $\zeta(\cdot)$ is uniform, proving (2).

We now prove (3) by showing $\zeta(0) \equiv \eta\left(s_{0}\right)$ for $b \gg 0$ (the other endpoint being similar). Exactly as in the claim at the start of the proof of Lemma 6.16, it is sufficient to prove that if $E$ is torsion-free of topological type $\tau$, and $F \subset E$ is saturated with $F \in \mathcal{S}_{K}$ it holds that

$$
p_{F \subset E}^{\eta\left(s_{0}\right)}(\leq) 0 \text { if and only if } p_{F \subset E}^{\zeta(0)}(\leq) 0 .
$$

To prove this, with $u_{i}(s)$ defined as in (6.19) one computes using Riemann-Roch (6.11) that if $F \subset E$, then

where

$$
p_{F \subset E}^{\zeta(r)}=\left\langle\left\langle u_{1}\left\|u_{2}(\bar{s})\right\| u_{3}(\bar{s})+\delta(r)\right\rangle\right\rangle
$$

$$
\delta(r)=\sum_{j} \operatorname{rank}\left(B_{j}(\bar{s})\right) \operatorname{hilb}_{1}(F, E) \cdot\left[(1-r) \operatorname{ch}_{2}\left(C_{j 0}\right)+r \operatorname{ch}_{2}\left(C_{j 1}\right)\right] .
$$

After some manipulation with (6.19) and (6.23), we in fact have

$$
\delta(r)=b\left[\lambda u_{1}+(1-r) u_{2}\left(s_{0}\right)+r u_{2}\left(s_{1}\right)-u_{2}(\bar{s})\right] .
$$

Thus, in particular

$$
p_{F \subset E}^{\zeta(0)}=\left\langle\left\langle u_{1}\left\|u_{2}(\bar{s})\right\| u_{3}(\bar{s})+b\left(u_{2}\left(s_{0}\right)-u_{2}(\bar{s})-\lambda u_{1}\right)\right\rangle\right\rangle .
$$

Now, clearly $p_{F \subset E}^{\zeta(0)}(\leq) 0$ if and only if

$$
\left\langle\left\langle u_{1}\left\|u_{2}(\bar{s})\right\| u_{3}(\bar{s})+b\left(u_{2}\left(s_{0}\right)-u_{2}(\bar{s})\right\rangle\right\rangle(\leq) 0,\right.
$$

which in turn occurs if and only if

$$
\left\langle\left\langle u_{1}|| u_{2}(\bar{s}) \| u_{3}(\bar{s})+b u_{2}\left(s_{0}\right)\right\rangle\right\rangle(\leq) 0 .
$$

Final Claim: For $b$ sufficiently large, the inequality (6.28) holds if and only if

$$
\left\langle\left\langle u_{1}\left\|u_{2}\left(s_{0}\right)\right\| u_{3}\left(s_{0}\right)\right\rangle\right\rangle(\leq) 0 .
$$

The argument for this is similar to what has been done before, cf. the proof of Lemma 6.16. Assume (6.28) holds. Then, $u_{1} \leq 0$. If this is strict we are done. So, we may assume $u_{1}=0$, and thus $u_{2}(\bar{s}) \leq 0$. If this inequality is strict, then as $s_{0}$ is in an adjacent chamber to $\bar{s}$ we 
have $u_{2}\left(s_{0}\right)<0$ and we are done. Otherwise $u_{2}(\bar{s})=0$ and $u_{3}(\bar{s})+b u_{2}\left(s_{0}\right) \leq 0$. As $b$ is large, this implies $u_{2}\left(s_{0}\right) \leq 0$. Again, if this is strict, we are done. Otherwise

$$
u_{2}\left(s_{0}\right)=0 \text { and } u_{3}(\bar{s})(\leq) 0 .
$$

By Lemma 6.25 below, the first of these statements gives hilb $1(F, E) c_{1}\left(L_{j}\right)^{2}=0$ and also $\operatorname{hilb}_{2}(F, E) c_{1}\left(L_{j}\right)=0$ for $j=0,1$. Hence, from (6.19), (6.11), and (6.16) we actually have $u_{3}(s)=h_{3}(\bar{t})$, which is independent of $s$. Consequently, from the second statement in (6.29) we deduce $u_{3}\left(s_{0}\right)(\leq) 0$ as well, proving one direction of the claim.

For the converse, assume $\left\langle\left\langle u_{1}|| u_{2}\left(s_{0}\right) \| u_{3}\left(s_{0}\right)\right\rangle\right\rangle \leq 0$. Then, $u_{1} \leq 0$, and if strict inequality holds we are done. Otherwise, $u_{1}=0$ and $u_{2}\left(s_{0}\right) \leq 0$, and so $u_{2}(\bar{s}) \leq 0$ by continuity. If strict inequality holds, then $u_{2}\left(s_{0}\right)<0$ as well, since $s_{0}$ is in an adjacent chamber, and we are done. So, we may assume $u_{2}(\bar{s})=0$. We divide into two cases. In the first case $u_{2}\left(s_{0}\right)=0$. Then, $u_{3}\left(s_{0}\right)(\leq) 0$ and hence by continuity $u_{3}(\bar{s})(\leq) 0$ (the case of equality here uses that $s_{0}$ lies in an open chamber, so $u_{3}\left(s_{0}\right)=0$ implies $\left.u_{3}(\cdot) \equiv 0\right)$. In the second case $u_{2}\left(s_{0}\right)<0$. But for $b$ large this implies $u_{3}(\bar{s})+b u_{2}\left(s_{0}\right)<0$, and we are done here as well. This proves the Final Claim, and hence concludes the proof of Lemma 6.19.

Lemma 6.25. Let $E$ be of topological type $\tau$ and $F \in \mathcal{S}_{K}$. With $u_{i}$ defined as in (6.19), suppose that $u_{1}=0$ and $u_{2}\left(s_{0}\right)=0$. Then, $u_{2} \equiv 0$, hilb $\operatorname{hi}_{1}(F, E) c_{1}\left(L_{j}\right)^{2}=0$, and

$$
\operatorname{hilb}_{2}(F, E) c_{1}\left(L_{j}\right)=0
$$

for $j=0,1$.

Proof. As $s_{0}$ is in an open chamber, the second assumption implies $u_{2} \equiv 0$. But looking back at (6.19) this implies $h_{1} \equiv 0$, which gives hilb $(F, E) c_{1}\left(L_{j}\right)^{2}=0$ for $j=0,1$ by (6.14). Looking again at (6.19) the fact that $u_{2} \equiv 0$ now implies $h_{2}(\bar{t})=0$. But by the assumption that $L_{0}$ and $L_{1}$ are separated by a single wall of the first kind (and not by any walls of the second kind) this is impossible unless $\operatorname{hilb}_{2}(F, E) c_{1}\left(L_{j}\right)=0$ for $j=0,1$ as well.

Remark 6.26. We mention again that it is the proof of Lemma 6.25 where the assumption on the two line bundles $L_{0}$ and $L_{1}$ being separated by a single wall of the first kind is used.

Proof of Lemma 6.20. We end this ordeal by proving that for $b$ sufficiently large $\zeta(\cdot)$ is open. As $\mathcal{S}_{K}$ detects the change of stability for $\zeta(\cdot)$, by Lemma 6.7 it is sufficient to prove that for $r$ sufficiently small, if $E$ is torsion-free of type $\tau$ and $F \subset E$ is saturated with $F \in \mathcal{S}_{K}$, then

$$
p_{F \subset E}^{\zeta(0)}(\leq) 0 \text { if and only if } p_{F \subset E}^{\zeta(r)}(\leq) 0
$$

and

$$
p_{F \subset E}^{\zeta(1)}(\leq) 0 \text { if and only if } p_{F \subset E}^{\zeta(1-r)}(\leq) 0 .
$$

We will prove the first of these, the second being proved in precisely the same way. So let $E$ and $F$ be such sheaves. Recall from (6.26), (6.27) that

$$
p_{F \subset E}^{\zeta(r)}=\left\langle\left\langle u_{1}\left\|u_{2}(\bar{s})\right\| u_{3}(\bar{s})+\delta(r)\right\rangle\right\rangle
$$

with

$$
\delta(r)=b\left[\lambda u_{1}+(1-r) u_{2}\left(s_{0}\right)+r u_{2}\left(s_{1}\right)-u_{2}(\bar{s})\right] .
$$

Since $u_{1}$ and $u_{2}(\bar{s})$ are independent of $r$, it is sufficient to show the following: if $u_{1}=u_{2}(\bar{s})=0$ and $u_{3}(\bar{s})+\delta(0)=0$, then $u_{3}(\bar{s})+\delta(\cdot) \equiv 0$.

So, assume $u_{1}=u_{2}(\bar{s})=0=u_{3}(\bar{s})+\delta(0)$. Then, the first equality implies $\delta(0)=b u_{2}\left(s_{0}\right)$, and so

$$
0=u_{3}(\bar{s})+b u_{2}\left(s_{0}\right)
$$


Since $b$ is arbitrarily large, this implies $u_{2}\left(s_{0}\right)=0$. Hence, the linear function $s \mapsto u_{2}(s)$ vanishes at the two distinct points $s=\bar{s}$ and $s=s_{0}$, and thus $u_{2}(s)=0$ for all $s \in[0,1]$. In particular, $u_{2}\left(s_{1}\right)=0$, and so the expression $u_{3}(\bar{s})+\delta(r)$ is actually independent of $r$, which completes the proof.

\section{REFERENCES}

[Ber14] Aaron Bertram, Stability and Positivity, talk given as part of "Positivity of Linear Series and Vector Bundles", workshop at Banff International Research Station, Jan. 2014, available online at www . birs.ca/events/2014/5-day-workshops/14w5056/videos

[DH98] Igor Dolgachev and Yi Hu, Variation of geometric invariant theory quotients, Inst. Hautes Études Sci. Publ. Math. 87 (1998), 5-56.

[GRT14] Daniel Greb, Julius Ross, and Matei Toma, Variation of Gieseker Moduli Spaces via Quiver GIT, I, preprint (To appear in Geometry and Topology), arXiv:1409.7564, 2014.

[GRT15] Daniel Greb, Julius Ross, and Matei Toma, Semi-continuity of Stability for Sheaves and Variation of Gieseker Moduli Spaces, preprint arXiv:1501.04440, 2015.

[GT13] Daniel Greb and Matei Toma, Compact moduli spaces for slope-semistable sheaves, preprint, arXiv: 1303.2480, 2013.

[Gro95] Alexander Grothendieck, Techniques de construction et théorèmes d'existence en géométrie algébrique. IV. Les schémas de Hilbert, Sém. Bourbaki, Vol. 6, Exp. No. 221, Soc. Math. France, Paris, 1995, pp. 249-276.

[HL10] Daniel Huybrechts and Manfred Lehn, The geometry of moduli spaces of sheaves, second ed., Cambridge Mathematical Library, Cambridge University Press, Cambridge, 2010.

[JII] Dominic Joyce Configurations in abelian categories. II. Ringel-Hall algebras, Adv. Math. 210 (2007), no. 2, 635-706.

[MW97] Kenji Matsuki and Richard Wentworth, Mumford-Thaddeus principle on the moduli space of vector bundles on an algebraic surface, Internat. J. Math. 8 (1997), no. 1, 97-148.

[Sch00] Alexander Schmitt, Walls for Gieseker semistability and the Mumford-Thaddeus principle for moduli spaces of sheaves over higher dimensional bases, Comment. Math. Helv. 75 (2000), no. 2, 216-231.

[Tha96] Michael Thaddeus, Geometric invariant theory and flips, J. Amer. Math. Soc. 9 (1996), no. $3,691723$.

DG: Essener Seminar für Algebraische Geometrie und Arithmetik, Fakultät für Mathematik, Universität Duisburg-Essen, 45117 Essen, Germany

E-mail address: daniel.greb@uni-due.de

JR: Department of Pure Mathematics and Mathematical Statistics, University of Cambridge, Wilberforce Road, Cambridge, CB3 0WB, UK

E-mail address: j.ross@dpmms.cam.ac.uk

MT: Institut de Mathématiques Élie Cartan, Université de Lorraine, B.P. 70239, 54506 Vandoeuvre-lès-Nancy Cedex, France

E-mail address: Matei.Toma@univ-lorraine.fr 